\title{
Oral herbal medicine for women with intrahepatic cholestasis in pregnancy: a systematic review of randomized controlled trials
}

Ruiting Wang ${ }^{1 \dagger}$, Nuo Cheng ${ }^{2 \dagger}$, Rongyan Peng ${ }^{2}$, Zeyu $\mathrm{Yu}^{3}$, Mengdie Nan ${ }^{1}$ and Huijuan Cao ${ }^{3 *}$ (D

\begin{abstract}
Background: Intrahepatic cholestasis of pregnancy (ICP) is a pregnancy complication whose range has been calculated to be between 0.01 and $15.6 \%$ all around the world. We wanted to systematically evaluate the effect and safety of oral herbal medicine on treatment for ICP.

Methods: Details of the methods could be found in the registered protocol on PROSPERO (CRD42018096013). Trials assessing the effectiveness of herbal medicine for ICP were searched from seven electronic databases from inception to 28th February 2020. RevMan 5.3 software was used to perform all statistical analysis. Meta-analysis, additional analysis, Trial Sequential Analysis (TSA) and Grading of Recommendations Assessment, Development and Evaluation (GRADE) were conducted if data permitted.

Results: Totally 43 randomized controlled trials with 3556 patients were included. Meta-analysis showed potential good adjunctive effect of herbal medicine on decreasing the pruritus scores (MD $-0.58,95 \% \mathrm{Cl}-0.79$ to -0.36$)$, the serum TBA scores (MD $-3.99 \mu \mathrm{mol} / \mathrm{L}, 95 \% \mathrm{Cl}-4.24$ to -3.74$)$ on the basis with Ursodesoxycholic acid. Compared to the medicine alone, significantly lower incidence of fetal distress (RR $0.41,95 \% \mathrm{Cl} 0.32$ to 0.51 ), asphyxia neonatorum (RR $0.35,95 \% \mathrm{Cl} 0.25$ to 0.49 ), cesarean section (RR $0.73,95 \% \mathrm{Cl} 0.63$ to 0.85 ), postpartum hemorrhage (RR $0.45,95 \% \mathrm{Cl} 0.28$ to 0.72 ) were observed in the combination group. But the comparison between herbal medicine and medicine showed inconsistent results among trials. Insufficient information could be used to evaluate the safety of herbal medicine for ICP.

Conclusion: This review found the current evidence may support the effectiveness of combination of herbal medicine and conventional medicine for decreasing the maternal pruritus scores, the serum TBA, and the number of fetal distress, or asphyxia neonatorum events related to this condition (which was supported by TSA results). Since there were obvious statistical and clinical heterogeneity among trials, and the methodological quality of the included studies was poor, the level of the evidence could only be defined as "very low" according to the GRADE criteria. Further high quality studies are still needed to testify the effectiveness and safety of herbal medicine for ICP.
\end{abstract}

Keywords: Herbal medicine, Intrahepatic cholestasis, Meta-analysis, Pregnancy, Systematic review

\footnotetext{
*Correspondence: huijuancao327@hotmail.com

${ }^{\dagger}$ Ruiting Wang and Nuo Cheng contributed equally to this work.

${ }^{3}$ Centre for Evidence Based Chinese Medicine, Beijing University of Chinese Medicine, Beijing, China

Full list of author information is available at the end of the article
} 


\section{Background}

Intrahepatic cholestasis of pregnancy (ICP) is a pregnancy complication with prevalence ranging between 0.01 and $15.6 \%$ [1-4]. ICP presents as persistent pruritus, typically on the skin of palms and soles, with elevated bile acid levels, and resolves with delivery [5]. Generally, the onset of ICP occurs in the second and third trimesters and disappears spontaneously after delivery, usually within weeks [5-7]. The clinical importance of ICP lies in the potential fetal risks, including spontaneous preterm birth, iatrogenic preterm birth and fetal death [8]. While the pathophysiology of ICP is still poorly understood, gene, hormone, and environment play roles. Bile acids need to enter hepatocytes or bile ducts through the action of some transporters which genetic mutations can cause cholestasis $[9,10]$. Estrogen can decrease the expression of ABCB11 / BSEP gene to inhibit the function of bile salt delivery pump, or decrease the activity of $\mathrm{Na}+/ \mathrm{K}+$ ATPase to inhibit the uptake of bile acid by hepatocytes that eventually leads to intrahepatic cholestasis. Sulfated progesterone metabolites can also have an adverse effect on bile acid excretion by inhibiting the function of BSEP [11]. Although the mechanism is unknown, studies have shown that the plasma and serum selenium concentrations and glutathione peroxidase activities in ICP patients are lower than those in healthy pregnant women, and the incidence rate is higher in winter. Some environmental factors such as pesticide pollutants, erucic acid in rape and selenium deficiency in food may lead to ICP [12]. Most often the disease affects women over the age of 35 years, [13] with personal history of cholestasis associated with the use of oral contraceptives, personal or family history of cholestasis of pregnancy, [14] biliary disease, [1] or liver disease, in multiple gestation pregnancy, [15] or in vitro fertilisation pregnancies [16]. Likewise, seasonal variations, [17] low selenium intake, erucic acid, increased gut absorption of bacterial endotoxins, pollutants, infections, and medicine are factors suspected as causing the disease [15-20].

When treating the ICP, doctors always focused on reducing maternal symptoms, improving results of liver tests, and reducing total bile acid (TBA) concentration. They commonly used Ursodeoxycholic acid (UDCA), Sadenosylmethionine (SAMe), dexamethasone, or cholestyramine as well as vitamin $\mathrm{K}$ (preventing postpartum bleeding) as therapies [20]. Whlie there was insufficient evidence to recommend early-term delivery or to support therapies above according to one Cochrane review [21]. However, the review found that UDCA seemed to improve the maternal symptom of pruritus, which agreed with the result of a meta-analysis by Bacq [22]. Bacq strongly suggested that UDCA was also beneficial for the fetal outcome, including total prematurity, fetal distress and neonatal respiratory distress syndrome; however, the Cochrane Review did not agree [21].

Some herbal medicine, such as Emodin, [23-25] Bushen Granule and Bushen Rougan Recipe, which includes Biejia (shell of Trionyx sinensis Wiegmann), Ejiao (made from skins of Equus asinus L.), Dihuang (tuberous root of Rehmannia glutinosa Libosch.), Gouqi (fruit of Lycium barbarum L.), Beishashen (root of Glehnia littoralis Fr. Schmidtex Miq.), Maidong (tuberous root of Ophio pogon japonicas (L. f) KerGawl.), Danggui (root of Angelica sinensis (Oliv.) Diels), Taoren (seed of Prunus persica (L.) Batsch), Qiancao (root of Rubia cordifolia L.), Baishao (root of Paeonia lactiflora Pall.), Huangjing (tuberous root of Polygonatum kingianum Coll. et Hemsl.), Jineijin (gizzard endothelium of Gallus Gallus domesticus), Dilong (Pheretima asperigillum), Haipiaoxiao (inner shell of Sepiella maindroni de Rochebrune), $[26,27]$ and in vivo cultured Calculus Bovis (made from bile of Bos taurus domesticus Gmelin) [28] were proved to be protective on cholestatic hepatitis by decreasing the levels of alanine aminotransferase (ALT) and total bile acid (TBA), prevent toxic compounds overaccumulation in hepatocytes. Decoctions like Zhi-Zi-Da-Huang decoction (including Zhizi (fruit of Gardenia jasminoides Ellis), Dahuang (Rheum palmatum L.), Zhishi (fruit of Citrus aurantium L.), Dandouchi (made from seeds of Glycine $\max$ (L.) Merr.) [29] and Shuangcao Tuihuang Granule-1 (including Yinchen (Artemisia scoparia Waldst. et Kit. Or Artemisia capillaries Thunb.), Huhuanglian (tuberous root of Picrorhiza scrophulariiflora Pennell), Tuxiangru (Origanum vulgare L.), Cheqianzi (Plantago asiatica L. or Plantago depressa Willd.), Shengdihuang (tuberous root of Rehmannia glutinosa Libosch.), Houpo (bark of Magnolia officinalis Rehd. et Wils.), etc.) [30] may also significantly dose-dependently reduce the indices of liver injuries by raising Superoxide Dismutase activity, scavenging oxygen free radicals and increasing anti-oxidation [28-30].

With the possible underlying mechanism of herbal medicinal for this condition, we conducted this systematic review to explore the potential effectiveness and safety of oral administration of herbal medicine in treating ICP.

\section{Methods}

\section{Protocol registration}

Protocol of this review was registered in PROSPERO as Ruiting Wang, Rongyan Peng, Nuo Cheng, Zeyu Yu, Mengdie Nan, Huijuan Cao. Oral herbal medicine for women with intrahepatic cholestasis in pregnancy: a systematic review of randomized controlled trial. PROSPERO 2018 CRD42018096013. Available from: http:// www.crd.york.ac.uk/PROSPERO/display_record.php?ID= CRD42018096013. 


\section{Eligibility criteria}

Published and unpublished studies, inany language, were included where thefollowing PICOS (patient, intervention, comparator, outcome, study type) criteria were met:

i) Patient: Patients, of any age, with intrahepatic cholestasis in pregnancy. Intrahepatic cholestasis in pregnancy (ICP or OC), diagnosed in accordance with recognized criteria (e.g. Guidelines for diagnosis and treatment of intrahepatic cholestasis in pregnancy (2015) by Chinese Medical Association). ii) Intervention: Herbal medicine through oral administration. iii) Comparators: Controls include placebo and conventional medicine (such as UDCA, SAMe, dexamethasone, vitamin $\mathrm{K}$ etc.). Chinese medicine combined with conventional medicine compared to the conventional medicine alone are also included. The controls in protocol were selected as Guidelines for diagnosis and treatment of intrahepatic cholestasis in pregnancy (2015) by Chinese Medical Association. After analyzing the data, most of the controls were not reported in the study, so there are some differences. iv) Outcomes: Primary outcomes included the changes in Ribalta score, maternal serum TBA values, and incidence of adverse birth events (e.g. newborn deaths, fetal distress and suffocation). Secondary outcomes included changes in bile acid content, incidence of cesarean sections, postpartum hemorrhage, adverse effects of medicine, changes in ALT values, and changes in AST values for the maternal, as well as the averages gestational age at birth and the incidence of premature births. v) Study type: Randomized controlled trials.

Literatures that unable to obtain the analysable data, as well as the piratical documents would be excluded.

\section{Searching strategy}

We searched seven databases and two clinical trial registration systems, including PubMed, SpringerLink, ProQuest, the Cochrane Central Register of Controlled Trials (CENTRAL), the Chinese National Knowledge Infrastructure Databases (CNKI), the Chongqing VIP Chinese Science and Technology Periodical Database (VIP), Wanfang Data Knowledge Service Platform, Chinese Clinical Trial Registry (ChiCTR), and Clinical Trials (ClinicalTrials.gov). The subject of the retrieval is: "Drugs, Chinese Herbal" [Mesh] OR "Herbal Medicine" [Mesh] OR "Herbal" [Mesh] with "Pregnancy" [Mesh] OR "Pregnancy Complications" [Mesh] OR "Infant" [Mesh] OR "Infant, Newborn" [Mesh] OR "Fetus" [Mesh] OR "Fetal Development" [Mesh] OR "Prenatal Diagnosis" [Mesh] OR "Fetal Monitoring" [Mesh] OR "Fetal Therapies" [Mesh] OR "Extraembryonic Membranes" [Mesh] OR "Placenta" [Mesh] OR "Placental Function Tests" [Mesh] OR "Uterine Monitoring" [Mesh] OR "Pelvimetry" [Mesh] OR "Oxytocics" [Mesh]
OR "Tocolytic Agents" [Mesh] OR "Tocolysis" [Mesh] OR "Maternal Health Services" [Mesh] OR "Peripartum Period" [Mesh] OR "Parity" [Mesh] OR "Perinatal Care" [Mesh] OR "Postpartum Period" [Mesh] OR "Labor Pain" [Mesh] OR "Obstetrical" [Mesh] OR "MaternalChild Nursing" [Mesh] OR "Midwifery" [Mesh] OR "Apgar Score" combined with "Cholestasis, Intrahepatic" [Mesh] AND "Random" to be adjusted for use in the different databases.

Meantime, the Chinese National Knowledge Infrastructure Databases (CNKI), Wanfang Data Knowledge Service Platform and ProQuest Dissertations were used to search for grey literature.

\section{Data extraction (selection and coding)}

Trials retrieved using the search strategy and those from additional sources were screened independently by two review authors to identify trials that potentially meet the inclusion criteria outlined above. The full texts of these trials were retrieved and independently assessed for eligibility by other two reviewers. Any disagreement was resolved through discussion with a third reviewer (Huijuan Cao). A predesigned form was used to extract data from the included trials for assessment of trial quality.

Extracted information included: i) General information: Including document number, title, first author, year(s) conducted, location (city, country), source, etc.; ii) Methodological related information: type of design, grouping number, random allocation method, Random concealment method, method of blinding, participants blinded, loss of follow up, report of selective outcome, calculation of sample size, baseline comparability; iii) Participants information: diagnostic criteria, inclusion criteria, exclusion criteria, source, sample size, age, gender, disease course. iv) Intervention information: Types of interventions, intervention performer, treatment duration; v) Outcome measures: Treatment outcomes: changes in Ribalta score, changes in TBA values, number of neonatal deaths, number of fetal distress or asphyxiation, changes in ALT values, changes in AST values, number of caesarean sections, postpartum hemorrhage, adverse medicine effects, average gestational age at birth, number of premature deliveries.

\section{Risk of bias assessment}

We assessed the methodological quality of the included trials using the risk of bias tool recommended by the Cochrane Collaboration (Higgins and Green, 2009). Seven elements were assessed: random sequence generation, allocation concealment, blinding of participants and personnel, blinding of outcome assessment, incomplete outcome data (according to record the missing data and the method to deal with it), selective reporting (determined by the consistency of the predefined and 
reported outcomes) and other bias (assessed according to sample size calculation, inclusion/exclusion criteria for participant recruitment, comparability of baseline data, funding sources).

\section{Strategy for data analysis}

All statistical analyses were performed using RevMan 5.3 (The Cochrane Collaboration) software. The results of each single trial were described. We presented results as risk ratio (RR) with its 95\% confidence intervals (CI) for dichotomous outcomes, and mean difference (MD) with 95\% CI for continuous outcomes. Statistical heterogeneity between the included trials were assessed using the $I^{2}$ value, and a meta-analysis were conducted if it had proved to be no significant clinical (relating to the participants, interventions, controls, and outcomes) and statistical heterogeneity ( $I^{2}$ values are less than $\left.75 \%\right)$ among the included trials. If the $I^{2}$ value was less than $25 \%$, a fixed-effect model (FEM) was used to synthesize the data, and if the $I^{2}$ value was between 25 and $75 \%$, we explored the sources of the heterogeneity. If the results of sensitive analysis or subgroup analysis explained the statistical heterogeneity successfully (i.e., $I^{2}$ value is less than 25\%), we used the FEM to synthesize the data as well, otherwise, random effect model (REM) was used to combine the data. Data would not be synthesized if there was a significant level of statistical heterogeneity (i.e., $I^{2}$ value is greater than 75\%) which was could not be explain or to handle (by subgroup analysis).

\section{Additional analysis}

If one meta-analysis included more than 10 trials, we explored the possibility of publication bias by using a funnel plot.

If one meta-analysis contained more than 8 included studies, we performed a Trial Sequential Analysis (TSA) was performed if there were more than 8 included studies in the meta-analysis. We calculated the required sample size of each meta-analysis and tested the robustness of the result with the TSA versioned 0.9.5.10 (Copenhagen: The Copenhagen Trial Unit, Center for Clinical Intervention Research, 2017). The information size required by the diversity-adjustment that we used was estimated from a control event proportion of the included trials and a priori intervention effect of $5 \%$, and the diversity we used was estimated in the included trials.

\section{Evidence quality assessment}

The Grading of Recommendations Assessment, Development and Evaluation criteria (GRADE) was conducted to assess the certainty of the evidence for each primary outcomes with meta-analysis. Considering the following aspects, such as study design, risk of bias, outcome consistency of trials, directness and accuracy of evidence and possibility of publication bias, we judged whether to downgrade the evidence. Finally, the evidence was rated at four levels: high, moderate, low or very low.

\section{Results}

\section{Selection results}

A total of 1604 trials were retrieved according to the search strategy, and the literature was screened according to the predefined criteria. After reading the title and abstract, 1262 trials were screened out, and further another 187 trials were excluded after reading the full text. Forty-three randomized controlled trials were finally included. All the included trials were published in Chinese. The specific literature screening process is shown in Fig. 1.

\section{Basic characteristics of trials (see Table 1)}

A total of 3556 women with ICP were included in the 43 trials [31-73]. The sample size ranged from 30 to 188 (an average of 41 patients in each group). The age distribution ranged from 18 to 42 years old, and the range of gestational age was $18-40$ weeks. All cases were recruited from the outpatient/inpatient Department of Obstetrics and Gynaecology. Thirty-eight trials reported baseline comparisons in terms of demographic characteristics, and the other five trials only mentioned randomization, which did not explain whether the baseline characteristic of the participants was comparable.

All patients were diagnosed according to the Chinese recognized criteria, including those cited in the textbook of "Obstetrics" [31, 32, 36-40, 59-65], the "Chinese obstetrics and gynaecology" [31, 34, 35, 41-50, 66-69], the "Obstetrics and Gynaecology Section of the Chinese Medical Association Obstetrics and Gynaecology Branch: Guide to diagnosis and treatment of intrahepatic cholestasis of pregnancy" [33, 51-53, 70], the "Practical Obstetrics and Gynaecology Handbook" [54], the "TCM Gynaecology" [45, 60, 65], the "Guidelines for diagnosis and treatment of intrahepatic cholestasis of pregnancy (2015)" [40, 71], the "Clinical obstetrics and gynaecology" [72], and the Reyes standard [73].

Among the included trials, four trials compared Chinese herbal medicine with conventional medicine [31, 32, 34, 35], 38 trials [34-73] compared the combination of the herbal medicine and conventional medicine with conventional medicine alone, the remaining one threearms trial [33] covered both of these two comparisons. As the intervention, 30 trials used herbal decoction with fixed prescriptions [33-35, 37-43, 45-53, 58, 60, 61, 65-69, 71-73], seven trials used self-made prescriptions according to syndrome differentiation principle [31, 44, 54-57, 63], six trials used herbal patent [32, 36, 59, 62, 64, 70]. Main herbal prescriptions (reported in two 


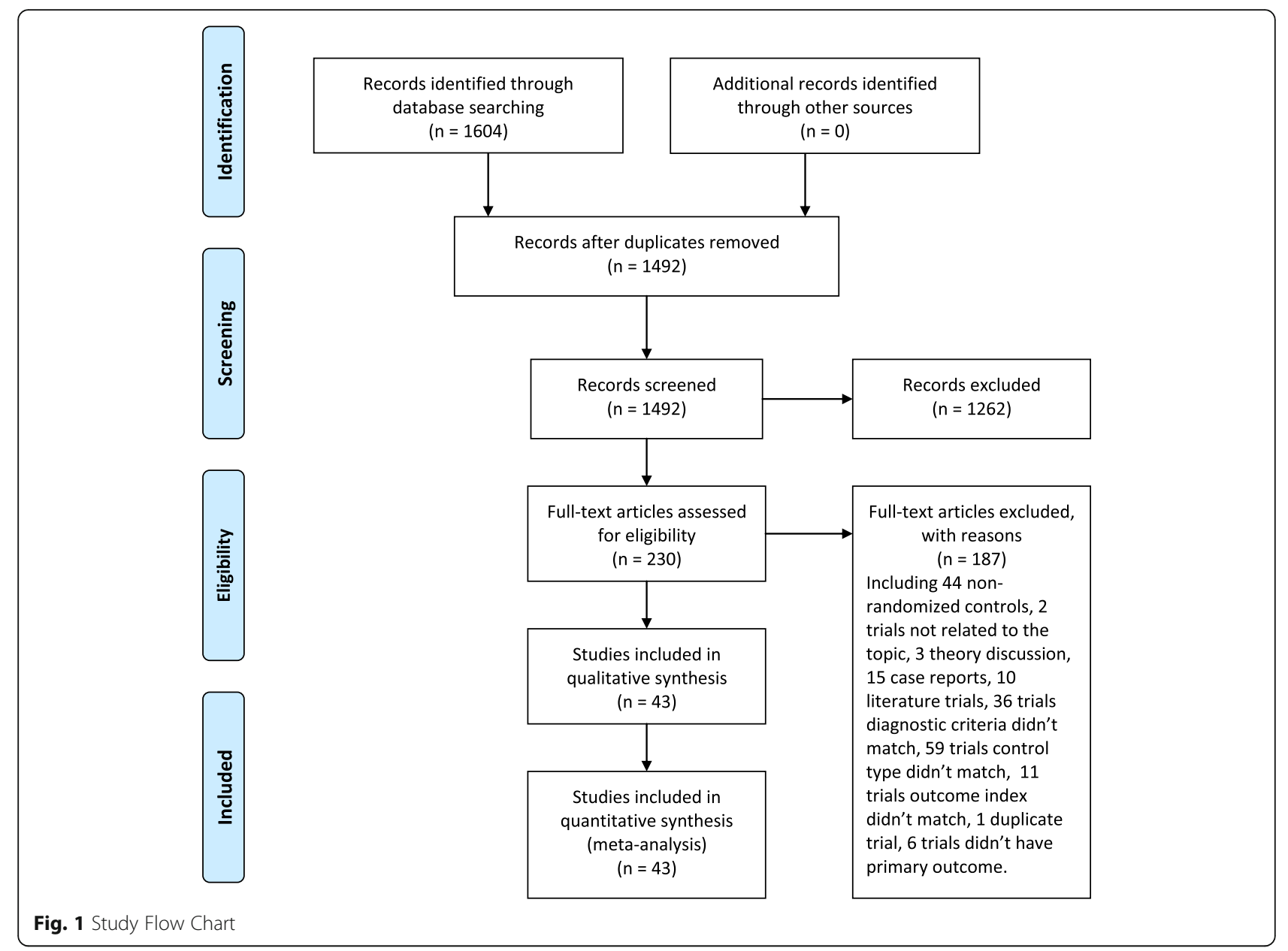

studies or more) was Yinchenhao Decoction $(n=7)$. Main herbs (reported in twenty studies or more) included: Yichen (Artemisiacapillaris thunb) $(n=38)$, Huangqin (Scutellaria baicalensis Georgi) $(n=29)$, Zhizi (Gardenia jasminoides Ellis) $(n=28)$, Dahuang (Rheum officinale Baill) $(n=21)$, Fuling (Poria cocos (Schw.) Wolf.) $(n=20)$. Herbal patents included Yinzhihuang oral liquid [32, 36, 59, 64], Yinzhihuang granule [62], Wuling pill [70]. The ingredients of them were showed in Supplementary material 1. Treatment durations varied from 7 to 21 days among the included trials.

Forty-three trials [31-73] used conventional medicine as control treatment, including Ursodesoxycholic acid (UDCA) [39, 42, 45, 53, 56, 58, 59, 61, 63, 64, 66, 68, 69] (P.O., $8 \sim 15$ $\mathrm{mg} / \mathrm{kg} / \mathrm{d}, 1 \sim 3$ times/d), S-adenosylmethionine (SAMe) [32, 34, 43, 44, 46, 48, 52, 70, 71, 73] (P.O., $1 \sim 2 \mathrm{~g} /$ d; or ivgtt., 0.8 $\sim 1 \mathrm{~g} / \mathrm{d}, 5 \%$ glucose injection $250 \mathrm{~mL}$ ), Dexamethasone [35] (P.O., $9 \mathrm{mg} / \mathrm{d}, 3$ times/d), Dexamethasone plus vitamin K3 [55], SAMe plus reduced glutathione (GSH) [31], UDCA plus SAMe [33, 36, 37, 51, 67], SAMe plus phenobarbital and dexamethasone [41, 49], Dexamethasone plus Phenobarbital [54], UDCA plus SAMe and dexamethasone [50, 57, 62], SAMe plus dexamethasone [38] (SAMe: ivgtt., $1.0 \mathrm{~g} / \mathrm{d}$; dexamethasone: i.m., $6 \sim 10 \mathrm{mg}, 1 \sim 2$ times/d), GSH plus UDCA and dexamethasone [47], UDCA plus SAMe plus magnesium isoglycyrrhizinate [60], UDCA plus phenobarbital plus SAMe [65], UDCA plus dexamethasone (for patients with tendency of premature birth) [40, 72].

Twenty-two trials reported the changes in the pruritus score [34-38, 41-44, 46-48, 50-53, 59, 66, 69-71, 73]. The pruritus score of 18 trials was based on the standard Ribalta pruritus score [34, 35, 37, 38, 41-44, 46, 48, 51, 53, 59, 66, 69-71, 73]. Twenty-seven trials reported the post-treatment TBA values [31-33, 36-41, 45-48, 50$55,57,58,60,63,66,67,71,73]$. Among them, 24 trials reported the serum TBA values [31-33, 36-41, 45, 46, $50-55,57,60,63,66,67,71,73]$. The TBA detection method of the remaining three studies is unknown [47, $48,58]$. One trial reported the changes in the serum TBA values after treatment [49]. Nine trials reported the neonatal death toll $[34,35,53,55,60,61,63,69,71]$. Twenty-seven trials reported the number of fetal distress $[31,34-38,40,41,43,45,47,49-51,53,60,62-65,67-$ 73]. Nineteen trials reported the number of asphyxia neonatorum [32, 37, 39-41, 43, 45, 47-53, 56, 61, 65, $67,71]$. Besides the above primary outcomes we defined 


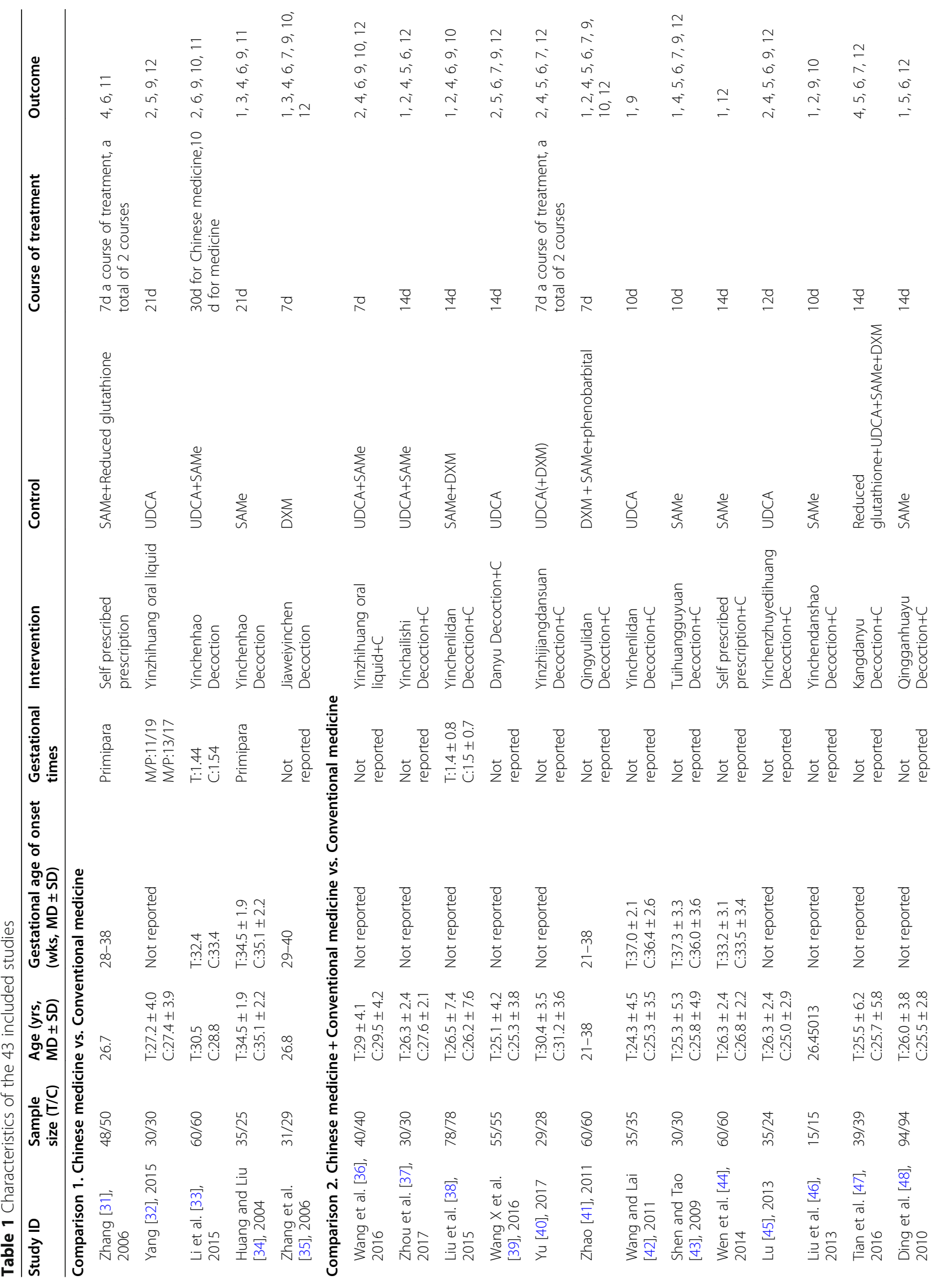



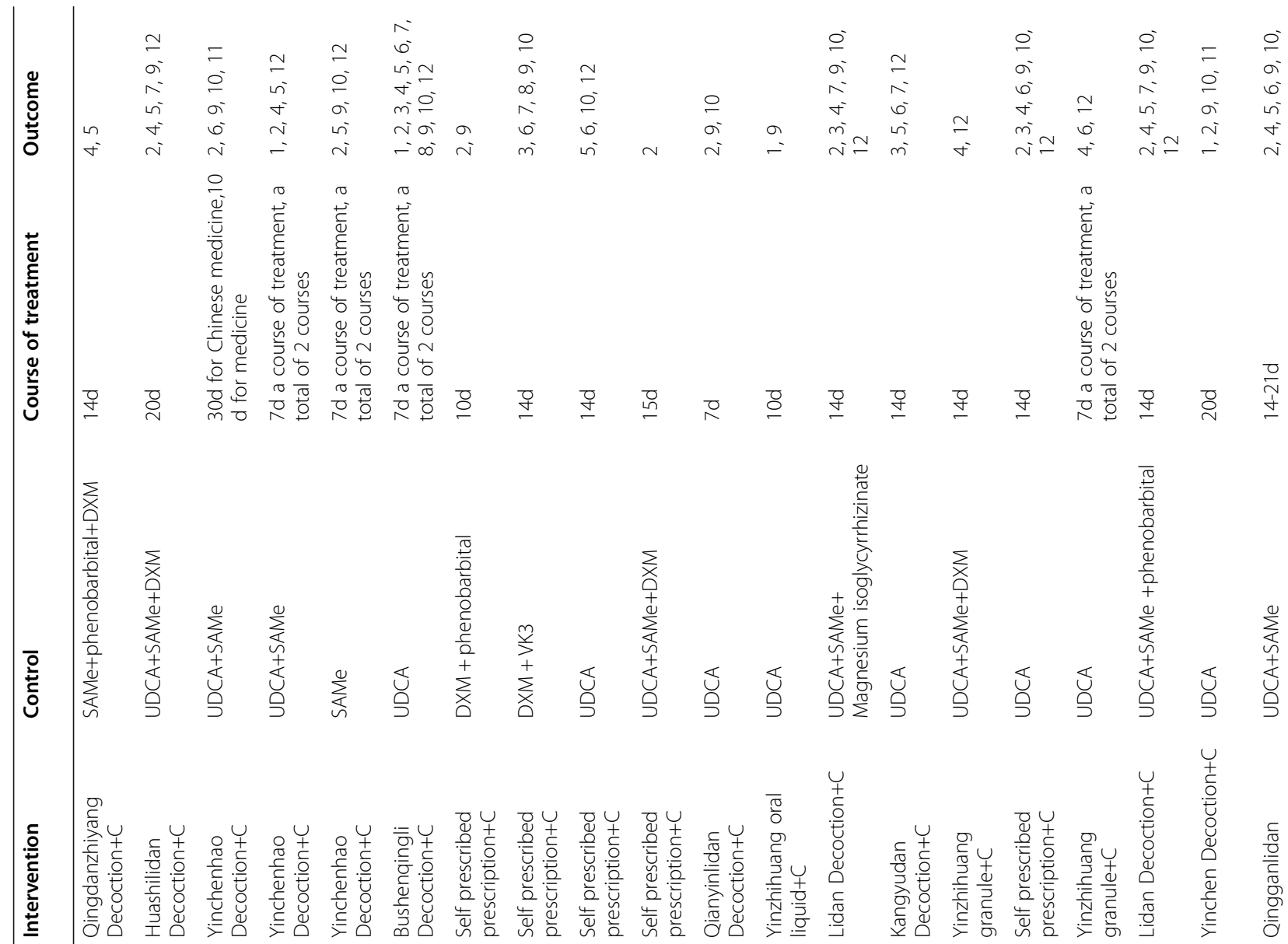

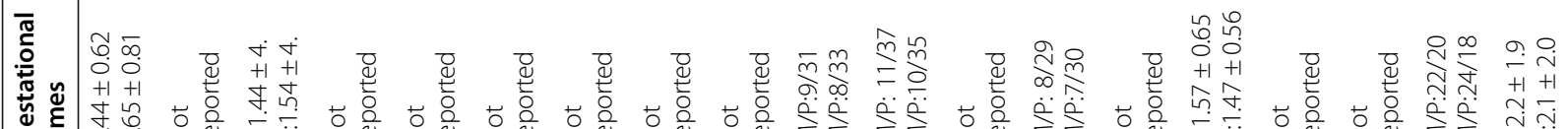

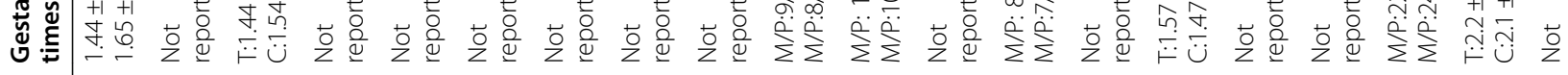

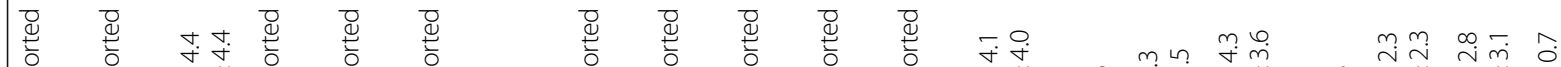

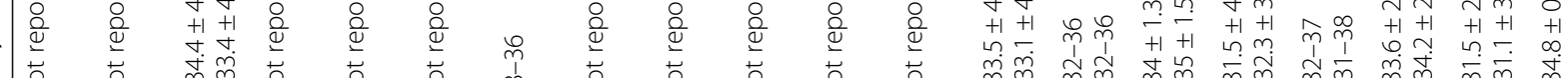

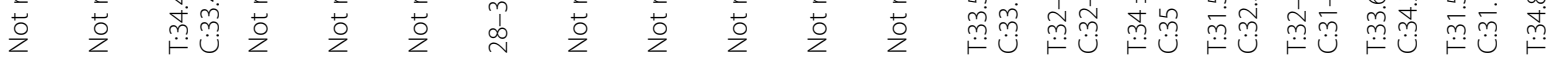

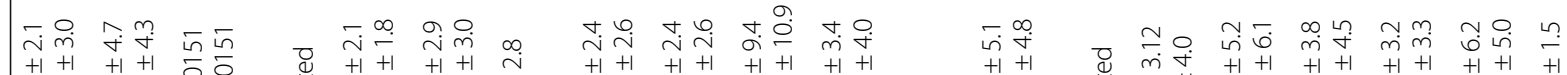

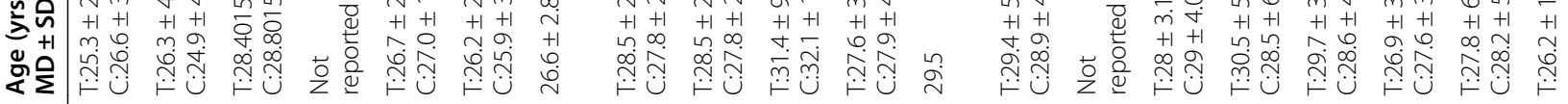

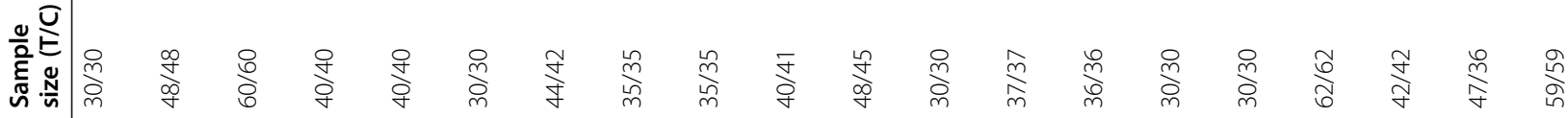

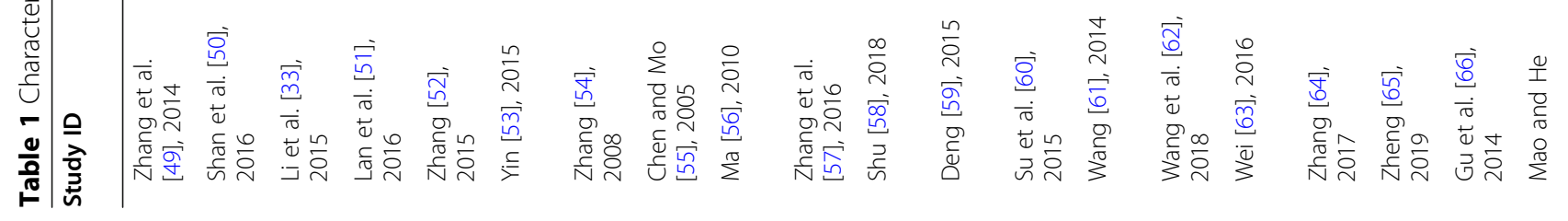


Wang et al. BMC Complementary Medicine and Therapies

(2020) $20: 303$

Page 8 of 24

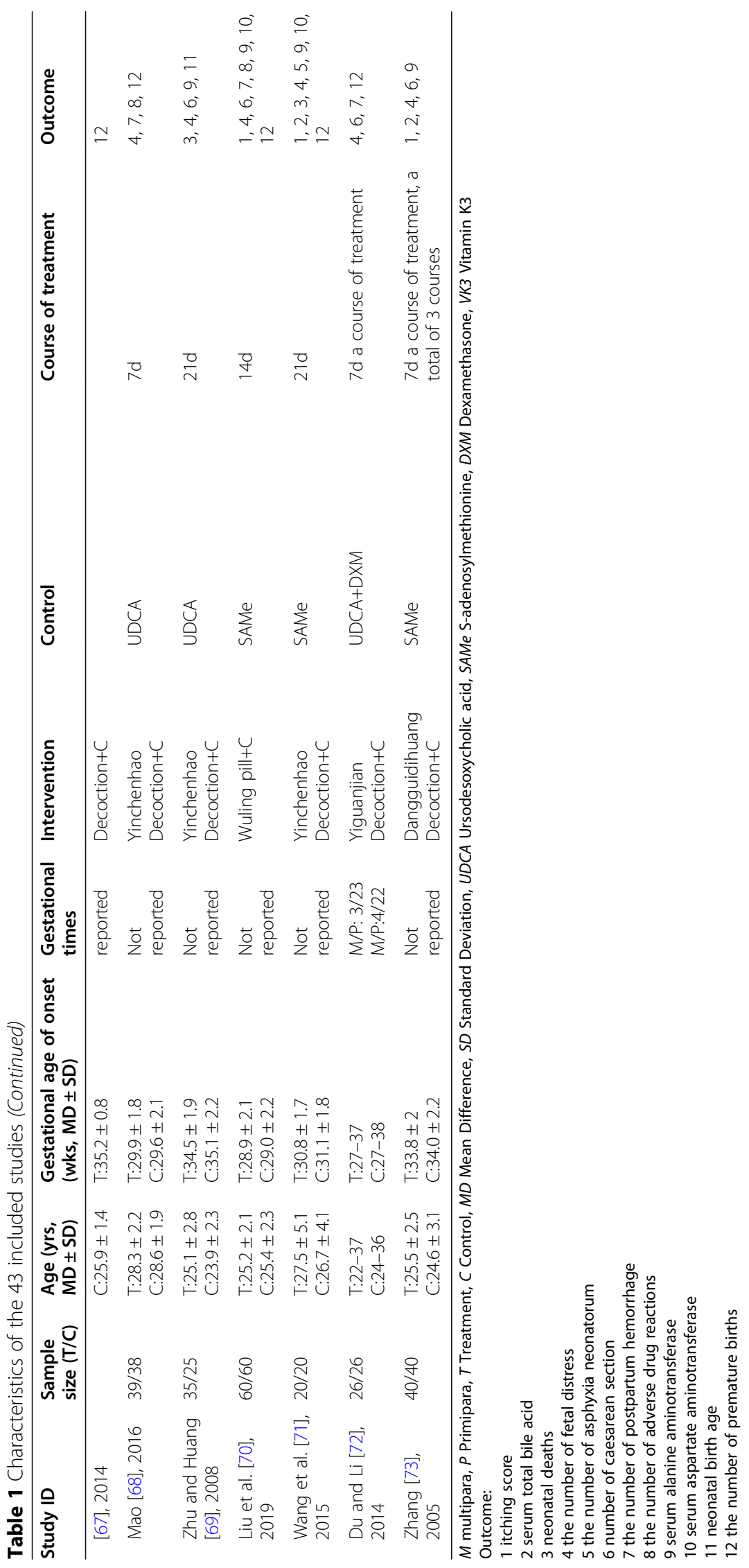


in this review, 25 trials reported the number of cesarean section $[31,33-41,43,45,47,48,53,55,56,61,63,64$, $67,69,70,72,73], 15$ trials reported the number of postpartum hemorrhage $[35,39-41,43,47,50,53,55,60$, $61,65,68,70,72]$, five trials reported the number of adverse events $[48,53,55,68,70]$. Twenty-nine trials reported the post-treatment serum ALT values [32-36, 38, $39,41-43,45,46,49-55,58-60,63,65-67,69-71,73]$, and 19 trials reported the post-treatment serum AST values $[33,35,36,38,41,46,49,50,52,53,55,58,60$, $63,65-67,70,71]$. Five trials reported the average gestational age of the newborn [31, 33, 34, 66, 69]. Twentyseven trials reported the number of premature birth [32, $35-37,39-41,43-45,47,48,50-55,60,62-65,67,68$, 70-72], and one trial reported the number of premature birth before gestational age of 37 weeks [45].

\section{Quality evaluation of inclusion studies}

None studies used the appropriate random number table method for sequence generation [32, 34, 38, 49, 57, 60, $68,69,71]$, one study used coinage method to generate random numbers [58]. It is unclear whether the remaining studies had used adequate randomization allocation methods. None of the 43 studies reported the allocation concealment or blinding of participants or investigators. One study reported and explained the dropouts and losses to follow-up [40], and none of the others reported dropouts. Two study has selective reporting outcomes [34, 51], because one of them intended to use liver function data as outcome measures but was not reported in result and another one reported the number of adverse birth events but was not reported in protocol. All studies did not report sample size estimates and financial support, also the baseline comparability was unclear. Thus, all the included trials were considered to be at high risk of other bias. So all the other bias risks of the 43 studies are unclear. In summary, all of the included trials were assessed as having high risk of bias due to the undefined methods of randomization, absence of blinding and the potential inappropriate method on dealing with missing data (See Fig. 2).

\section{Estimate effects (see Table 2)}

Herbal medicine vs. conventional medicine

A total of five studies compared herbal medicine with liver protection medicine [31-35].

Two studies reported changes in pruritus score of the pregnancy which was based on the standard Ribalta pruritus score [34, 35]. The analysis showed potential good adjunctive effect of herbal medicine on decreasing the pruritus scores on the basis with conventional medicine (MD $-0.68,95 \% \mathrm{CI}-0.88$ to $-0.47, I^{2}=0 \%, P<$ 0.00001, 2 trials, 120 patients). Two studies reported changes in maternal serum TBA [32,33], since the obvious statistical heterogeneity among studies $\left(I^{2}=95 \%\right)$, it was impossible to combine the data (MD and 95\% CI were $-4.74 \mu \mathrm{mol} / \mathrm{L},-6.57$ to -2.91 ; and $1.40 \mu \mathrm{mol} / \mathrm{L}$, 0.45 to 3.25 ; respectively). Two trials reported stillbirths or neonatal deaths post-treatment, and both two groups reported none $[34,35]$. Three studies reported the number of fetal distress [31, 34, 35], the results showed that there was no significant difference between the herbal medicine group and conventional medicine group (RR $0.77,95 \%$ CI 0.43 to $1.39, I^{2}=0 \%, P=0.36,3$ trials, 218 patients). Another study [32] reported the numbers of neonatal asphyxia, which also showed no difference between the herbal medicine and UDCA (RR 1.00, 95\% CI 0.06 to $16.76, P=1.00,1$ trial, 60 patients).

Four studies reported the number of deliveries by cesarean section [31,33-35], the results showed that there was no difference between herbal medicine and conventional medicine (RR $0.97,95 \%$ CI 0.80 to 1.18 , $I^{2}=0 \%, P=0.78,4$ trials, 338 patients). One trial reported the number of postpartum hemorrhage [35] and the results showed that there was no significant difference in this outcome between herbal medicine and conventional medicine (RR $0.47,95 \%$ CI 0.09 to $2.36, P=$ 0.36, 1 trial, 60 patients). One trial reported changes in maternal ALT (unknown as whole blood or serum) and the results showed that there was no significant difference in this outcome between herbal medicine and conventional medicine (MD $-12.80 \mathrm{U} / \mathrm{L}, 95 \% \mathrm{CI}-25.81$ to $0.21, P=0.05,1$ trial, 98 patients) [31]. Four studies reported changes in maternal serum ALT and the results

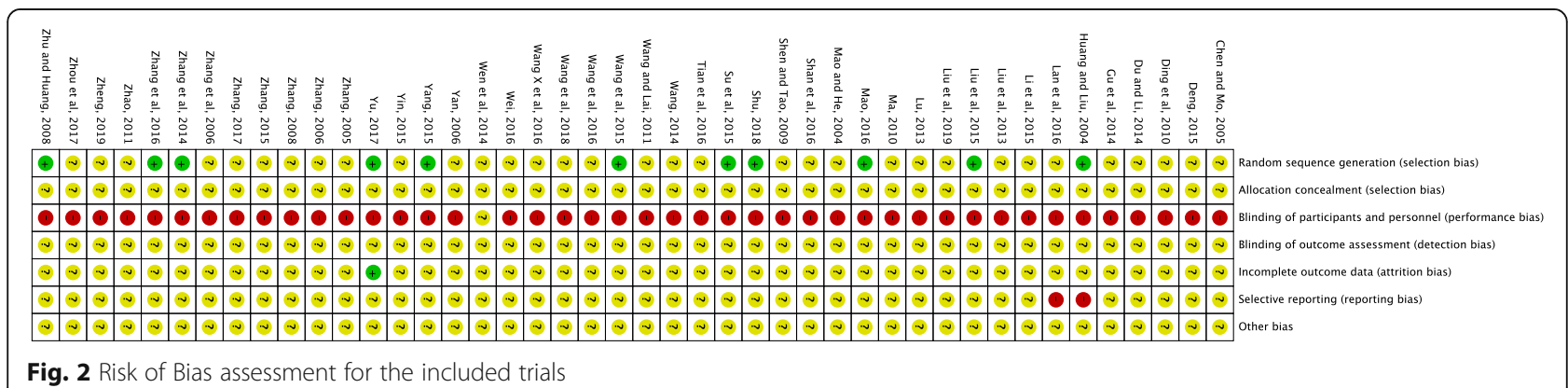

Fig. 2 Risk of Bias assessment for the included trials 
Table 2 Effect of estimates of oral herbal medicine for intrahepatic cholestasis in pregnancy in 43 included trials

\begin{tabular}{lll}
\hline Trials & Intervention/Comparison & $\begin{array}{l}\text { Effect estimates } \\
(95 \% \mathrm{Cl})\end{array}$ \\
\hline
\end{tabular}

\section{The pruritus score of the pregnancy}

1.1 Herbal medicine vs. Conventional medicine

Huang and Liu [34], 2004 Yinchenhao Decoction

Zhang et al. [35], 2006 Jiaweiyinchen Decoction

Subtotal (REM, $I^{2}=0 \%$ )

1.2 Herbal medicine plus Conventional

medicine versus Conventional medicine

1.2.1 Herbal medicine plus SAMe versus SAMe

Shen and Tao [43], 2009 Tuihuangguyuan Decoction

Wen et al. [44], $2014 \quad$ Self prescribed prescription

Liu et al. [46], $2013 \quad$ Yinchendanshao Decoction

Ding et al. [48], 2010 Qingganhuayu Decoction

Liu et al. [70], 2019

Wuling pill

Wang et al. [71], 2015

Yinchen Decoction

Zhang [73], 2005

Dangguidihuang Decoction

1.2.2 Herbal medicine plus UDCA versus UDCA

Wang and Lai [42], 2011 Yinchenlidan Decoction

Yin [53], 2015

Bushenqingli Decoction

Deng [59], 2015

Yinzhihuang oral liquid

Gu et al. [66], 2014

Yinchen Decoction

Subtotal (REM, $I^{2}=56 \%$ )

1.2.3 Herbal medicine plus UDCA plus SAMe versus UDCA plus SAMe

Zhou et al. [37], $2017 \quad$ Yinchailishi Decoction

Lan et al. [51], $2016 \quad$ Yinchenhao Decoction

Subtotal (REM, $I^{2}=95 \%$ )

1.2.4 Herbal medicine plus dexamethasone versus dexamethasone

Liu et al. [38], $2015 \quad$ Yinchenlidan Decoction

Zhao [41], 2011

Qingyulidan Decoction

Subtotal (FEM, $I^{2}=0 \%$ )

\section{Maternal serum TBA}

2.1 Herbal medicine vs. Conventional medicine
Yang [32], 2015
Yinzhihuang oral liquid vs. UDCA
Li et al. [14], 2015
Yinchenhao Decoction vs. UDCA+SAM

2.2 Herbal medicine plus Conventional medicine versus Conventional medicine

2.2.1 Herbal medicine plus SAMe versus SAMe

$\begin{array}{ll}\text { Liu et al. [46], 2013 } & \text { Yinchendanshao Decoction } \\ \text { Zhang [52], 2015 } & \text { Yinchenhao Decoction } \\ \text { Wang et al. [71], 2015 } & \text { Yinchen Decoction } \\ \text { Zhang [73], 2005 } & \text { Dangguidihuang Decoction }\end{array}$

Subtotal (FEM, $I^{2}=0 \%$ )

2.2.2 Herbal medicine plus UDCA versusUDCA

Wang $X$ et al. [39], 2016

Danyu Decoction

Yu [40], 2017

Yinzhijiangdansuan Decoction

Lu [45], 2013

Yinchenzhuyedihuang Decoction
$\mathrm{MD}-0.70[-0.93,-0.47]$

$\mathrm{MD}-0.55[-1.07,-0.03]$

MD $-0.68[-0.88,-0.47]<0.00001$
$\mathrm{MD}-4.74[-6.57,-2.91]$

MD $1.40[-0.45,3.25]$

MD $-10.66[-19.08,-2.24]$

MD $-8.20[-10.04,-6.36]$

$\mathrm{MD}-6.00[-8.18,-3.82]$

$\mathrm{MD}-7.20[-9.39,-5.01]$

MD - 7.33 $[-8.50,-6.15]<0.00001$

$\mathrm{MD}-6.44[-9.73,-3.15]$

$\mathrm{MD}-4.33[-7.48,-1.18]$

$\mathrm{MD}-3.51[-4.88,-2.14]$ 
Table 2 Effect of estimates of oral herbal medicine for intrahepatic cholestasis in pregnancy in 43 included trials (Continued)

\begin{tabular}{cl}
\hline Trials & Intervention/Comparison \\
\hline Yin [53], 2015 & Bushenqingli Decoction \\
Shu [58], 2018 & Qianyinlidan Decoction \\
Wei [63], 2016 & Self prescribed prescription \\
Gu et al. [66], 2014 & Yinchen Decoction \\
Subtotal (FEM, I² =0\%) & \\
2.2.3 Herbal medicine plus & UDCA plus SAMe versus UDCA plus SAMe \\
Wang et al. [36], 2016 & Yinzhihuang oral liquid \\
Zhou et al. [37], 2017 & Yinchailishi Decoction \\
Liu et al. [38], 2015 & Yinchenlidan Decoction \\
Lan et al. [51], 2016 & Yinchenhao Decoction \\
Su et al. [60], 2015 & Lidan Decoction \\
Mao and He [67], 2014 & Qingganlidan Decoction \\
Zheng [65], 2019 & Lidan Decoction
\end{tabular}

$\begin{aligned} & \text { Effect estimates } \\ & \text { (95\%Cl) }\end{aligned}$
MD -4.45 [-5.76, - 3.14]
MD -3.94 [-4.20,-3.68]
MD -5.00 [-6.78,-3.22]
MD -4.81 [-7.25,-2.37]

$\mathrm{MD}-2.30[-4.18,-0.42]$

$\mathrm{MD}-7.08[-9.88,-4.28]$

$\mathrm{MD}-8.13[-9.45,-6.81]$

$\mathrm{MD}-1.77[-4.30,0.76]$

$M D-3.42[-6.32,-0.52]$

MD $-24.10[-26.05,-$

22.15]

$\mathrm{MD}-6.38[-7.69,-5.07]$

MD - $7.62[-12.97$,

2.27]

MD-3.57 $[-4.74,-2.40]$
$M D-3.54[-5.33,-1.75]$
$M D-5.00[-6.71,-3.29]$
$M D-3.37[-3.86,-2.88]$

MD-3.50 $[-3.93,-3.08]$

$<0.00001$

RR $0.93[0.39,2.20]$

RR $0.86[0.29,2.50]$

$\operatorname{RR} 0.47[0.13,1.70]$

RR $0.77[0.43,1.39]$

Pvalue

0.005
MD-3.99 $[-4.24,-3.74] \quad<0.00001$

3.2 Herbal medicine plus Conventional medicine versus Conventional medicine
Wang et al. [36], 2016

Zhou et al. [37], 2017

Liu et al. [38], 2015

Yu [40], 2017

Zhao [41], 2011

Shen and Tao [43], 2009

Lu [45], 2013

Tian et al. [47], 2016

Zhang et al. [49], 2014

Shan et al. [50], 2016

Lan et al. [51], 2016

Yin [53], 2015

Su et al. [60], 2015
Yinzhihuang oral liquid plus UDCA and SAMe versus UDCA and SAMe Yinchailishi Decoction plus UDCA and SAMe versus UDCA and SAMe Yinchenlidan Decoction plus UDCA and SAMe versus UDCA and SAMe Yinzhijiangdansuan Decoction plus UDCA versus UDCA

Qingyulidan Decoction plus UDCA, SAMe and dexamethasone versusUDCA, SAMe and dexamethasone

Tuihuangguyuan Decoction plus SAMe versus SAMe Yinchenzhuyedihuang Decoction plus UDCA versus UDCA

Kangdanyu Decoction plus Reduced glutathione plus UDCA plus SAMe plus DXM versus Reduced glutathione plus UDCA plus SAMe

Qingdanzhiyang Decoction plus SAMe, phenobarbital and DXM versus SAMe, phenobarbital and DXM

Huashilidan Decoction plusUDCA, SAMe and dexamethasone versusUDCA, SAMe and dexamethasone

Yinchenhao Decoction plus UDCA and SAMe versus UDCA and SAMe Bushenqingli Decoction plus UDCA versus UDCA

Lidan Decoction plus UDCA, SAMe and ubtotal (FEM, $l^{2}=8 \%$ )

RR $0.22[0.05,0.96]$

RR 0.33 [0.01, 7.87]

RR 0.31 [0.12, 0.81]

RR $0.39[0.08,1.83]$

RR $0.25[0.06,1.13]$

RR $0.38[0.11,1.28]$

RR $0.29[0.08,1.02]$

$\operatorname{RR} 1.40[0.71,2.76]$

RR $0.27[0.08,0.88]$

$\operatorname{RR} 0.40[0.08,1.96]$

RR 0.54 [0.24, 1.21]

RR $0.33[0.10,1.11]$

RR $0.67[0.12,3.76]$ 
Table 2 Effect of estimates of oral herbal medicine for intrahepatic cholestasis in pregnancy in 43 included trials (Continued)

\begin{tabular}{|c|c|}
\hline Trials & Intervention/Comparison \\
\hline & $\begin{array}{l}\text { Magnesium isoglycyrrhizinate versus UDCA, SAMe and } \\
\text { Magnesium isoglycyrrhizinate }\end{array}$ \\
\hline Wang et al. [62], 2018 & $\begin{array}{l}\text { Yinzhihuang granule plus UDCA, SAMe and DXM versus UDCA, SAMe and } \\
\text { DXM }\end{array}$ \\
\hline Wei [63], 2016 & Self prescribed prescription plus UDCA versus UDCA \\
\hline Zhang [64], 2017 & Lidan Decoction plus UDCA versus UDCA \\
\hline Zheng [65], 2019 & $\begin{array}{l}\text { Yinchen Decoction plus UDCA, SAMe and Phenobarbital versus UDCA, } \\
\text { SAMe and Phenobarbital }\end{array}$ \\
\hline Mao and He [67], 2014 & Qingganlidan Decoction plus UDCA and SAMe versus UDCA and SAMe \\
\hline Mao [68], 2016 & Yinchenhao Decoction plus UDCA versus UDCA \\
\hline $\begin{array}{l}\text { Zhu and Huang [69], } \\
2008\end{array}$ & Yinchenhao Decoction plus UDCA versus UDCA \\
\hline Liu et al. [70], 2019 & Wuling pill plus SAMe versus SAMe \\
\hline Wang et al. [71], 2015 & Yinchenhao Decoction plus SAMe versus SAMe \\
\hline Du and Li [72], 2014 & Yiguanjian Decoction plus UDCA and DXM versus UDCA and DXM \\
\hline Zhang [73], 2005 & Dangguidihuang Decoction plus SAMe versus SAMe \\
\hline
\end{tabular}

Effect estimates

(95\% Cl)

RR $0.20[0.05,0.84]$

RR $0.21[0.08,0.55]$

RR $0.25[0.03,2.17]$

RR $0.50[0.05,5.31]$

RR $0.31[0.12,0.80]$

RR $0.97[0.06,15.02]$

$\operatorname{RR} 0.86[0.29,2.50]$

RR $0.40[0.08,1.98]$

Not estimable

RR $0.35[0.17,0.75]$

RR $0.50[0.21,1.20]$

RR0.41 [0.32, 0.51]

$<0.00001$

\section{The number of asphyxia neonatorum}

4.1 Herbal medicine vs. Conventional medicine

Yang [32], $2015 \quad$ Yinzhihuang oral liquid vs UDCA

RR $1.00[0.06,16.76]$

1.00

4.2 Herbal medicine plus Conventional medicine versus Conventional medicine

Zhou et al. [37], 2017

Wang $X$ et al. [39], 2016

Yu [40], 2017

Zhao [41], 2011

Shen and Tao [43], 2009

Lu [45], 2013

Tian et al. [47], 2016

Ding et al. [48], 2010

Zhang et al. [49], 2014

Shan et al. [50], 2016

Lan et al. [51], 2016

Zhang [52], 2015

Yin [53], 2015

Ma [56], 2010

Wang [61], 2014

Zheng [65], 2019

Mao and He [67], 2014

Wang et al. [71], 2015
Yinchailishi Decoction plus UDCA and SAMe versus UDCA and SAMe

Danyu Decoction plus UDCA versus UDCA

Yinzhijiangdansuan Decoction plus UDCA versus UDCA

Qingyulidan Decoction plus UDCA, SAMe and dexamethasone versusUDCA SAMe and dexamethasone

Tuihuangguyuan Decoction plus SAMe versus SAMe

Yinchenzhuyedihuang Decoction plus UDCA versus UDCA

Kangdanyu Decoction plus Reduced glutathione, UDCA, SAMe and DXM versus Reduced glutathione, UDCA and SAMe

Qingganhuayu Decoction plus SAMe versus SAMe

Qingdanzhiyang Decoction plus SAMe, phenobarbital and DXM versus SAMe, phenobarbital and DXM

Huashilidan Decoction plus UDCA, SAMe and dexamethasone versusUDCA, SAMe and dexamethasone

Yinchenhao Decoction plus UDCA and SAMe versus UDCA and SAMe

Yinchenhao Decoction plus SAMe versus SAMe

Bushenqingli Decoction plus UDCA versus UDCA

Self prescribed prescription plus UDCA versus UDCA

Kangyudan Decoction plus UDCA versus UDCA

Yinchen Decoction plus UDCA, SAMe and Phenobarbital versus UDCA, SAMe and Phenobarbital

Qingganlidan Decoction plus UDCA and SAMe versus UDCA and SAMe

Yinchenhao Decoction plus SAMe versus SAMe
RR $0.33[0.01,7.87]$

RR $0.33[0.07,1.58]$

RR $0.48[0.05,5.03]$

RR $0.25[0.03,2.17]$

RR $0.11[0.01,1.98]$

RR $0.29[0.08,1.02]$

RR $0.85[0.43,1.65]$

RR $0.33[0.13,0.88]$

RR 0.13 [0.02, 0.94]

$\operatorname{RR} 0.20[0.01,4.06]$

$\operatorname{RR} 0.50[0.19,1.33]$

RR $0.25[0.06,1.11]$

RR $0.13[0.02,0.94]$

RR $0.38[0.11,1.30]$

RR $0.40[0.08,1.93]$

RR $0.33[0.01,7.96]$

RR $0.23[0.07,0.77]$

Not estimable

RR0.35 [0.25, 0.49]
Pvalue 
Table 2 Effect of estimates of oral herbal medicine for intrahepatic cholestasis in pregnancy in 43 included trials (Continued)

\begin{tabular}{ll}
\hline Trials & Intervention/Comparison \\
\hline Zhang [31], 2006 & Self prescribed prescription vs SAMe and Reduced glutathione \\
Li et al. [14], 2015 & Yinchenhao Decoction vs. UDCA and SAM \\
Huang and Liu [34], 2004 & Yinchenhao Decoction vs SAMe \\
Zhang et al. [35], 2006 & Jiaweiyinchen Decoction vs DXM
\end{tabular}

\section{Overall (FEM, $I^{2}=0 \%$ )}

\section{Effect estimates \\ (95\% Cl) \\ RR $0.93[0.56,1.55]$ \\ RR $1.07[0.85,1.37]$ \\ RR $0.93[0.56,1.55]$ \\ RR $0.58[0.22,1.58]$}

RR $0.97[0.80,1.18]$

0.78

5.2 Herbal medicine plus Conventional medicine versus Conventional medicine

Wang et al. [36], 2016

Zhou et al. [37], 2017

Liu et al. [38], 2015

Wang $X$ et al. [39], 2016

Yu [40], 2017

Zhao [41], 2011

Shen and Tao [43], 2009

Lu [45], 2013

Tian et al. [47], 2016

Ding et al. [48], 2010

Li et al. [14], 2015

Yin [53], 2015

Chen and Mo [55], 2005

Ma [56], 2010

Wang [61], 2014

Wei [63], 2016

Zhang [64], 2017

Mao and He [67], 2014

Zhu and Huang [69],

2008

Liu et al. [70], 2019

Du and Li [72], 2014

Zhang [73], 2005

Overall (REM, $I^{2}=65 \%$ )
Yinzhihuang oral liquid plus UDCA and SAMe versus UDCA and SAMe Yinchailishi Decoction plus UDCA and SAMe versus UDCA and SAMe Yinchenlidan Decoction plus UDCA and SAMe versus UDCA and SAMe Danyu Decoction plus UDCA versus UDCA Yinzhijiangdansuan Decoction plus UDCA versus UDCA

Qingyulidan Decoction plus UDCA, SAMe and dexamethasone versus UDCA, RR $0.95[0.73,1.24]$ SAMe and dexamethasone

Tuihuangguyuan Decoction plus SAMe versus SAMe

Yinchenzhuyedihuang Decoction plus UDCA versus UDCA

Kangdanyu Decoction plus Reduced glutathione, UDCA, SAMe and DXM versus Reduced glutathione, UDCA and SAMe

Qingganhuayu Decoction plus SAMe versus SAMe

Yinchenhao Decoction plus UDCA andSAMe versus UDCA and SAMe

Bushenqingli Decoction plus UDCA versus UDCA

Self prescribed prescription plus DXM and VK3 versus DXM and VK3

Self prescribed prescription plus UDCA versus UDCA

Kangyudan Decoction plus UDCA versus UDCA

Self prescribed prescription plus UDCA versus UDCA

Lidan Decoction plus UDCA versus UDCA

Qingganlidan Decoction plus UDCA and SAMe versus UDCA and SAMe

Yinchenhao Decoction plus UDCA versus UDCA

Wuling pill plus SAMe versus SAMe

Yiguanjian Decoction plus UDCA and DXM versus UDCA and DXM

Dangguidihuang Decoction plus SAMe versus SAMe

RR $0.27[0.08,0.90]$

RR $0.69[0.53,0.90]$

RR $0.71[0.51,0.99]$

RR $0.38[0.23,0.62]$

RR $0.57[0.32,1.02]$

RR $0.91[0.67,1.24]$

RR $0.53[0.28,1.00]$

RR $0.52[0.30,0.89]$

RR $1.03[0.89,1.19]$

RR $0.80[0.59,1.08]$

RR $0.50[0.14,1.82]$

RR $1.08[0.83,1.39]$

RR $1.11[0.72,1.71]$

$\operatorname{RR} 0.67[0.46,0.97]$

RR $0.50[0.19,1.29]$

RR $0.27[0.08,0.93]$

RR $0.80[0.57,1.13]$

RR $0.93[0.56,1.55]$

RR $0.17[0.02,1.34]$

RR 0.95 [0.61, 1.49]
RR $0.39[0.20,0.77]$

RR $0.73[0.63,0.85]$

$<0.0001$

6. The number of postpartum haemorrhage

6.1 Herbal medicine plus versus Conventional medicine

Zhang et al. [35], 2006 Jiaweiyinchen Decoction vs DXM

RR $0.47[0.09,2.36]$

0.36

6.2 Herbal medicine plus Conventional medicine versus Conventional medicine

Wang $X$ et al. [39], 2016 Danyu Decoction plus UDCA versus UDCA

Yu [40], 2017

Zhao [41], 2011

Shen and Tao [43], 2009

Tian et al. [47], 2016

Shan et al. [50], 2016

Yin [53], 2015 SAMe and dexamethasone SAMe and dexamethasone
Yinzhijiangdansuan Decoction plus UDCA versus UDCA

RR $0.50[0.05,5.36]$

RR $0.48[0.05,5.03]$

RR $2.00[0.19,21.47]$

Tuihuangguyuan Decoction plus SAMe versus SAMe

Kangdanyu Decoction plus Reduced glutathione, UDCA, SAMe and DXM versus Reduced glutathione, UDCA and SAMe

$\operatorname{RR} 2.00[0.19,20.90]$

RR $0.25[0.06,1.10]$

$\operatorname{RR} 0.50[0.05,5.33]$

Bushenqingli Decoction plus UDCA versus UDCA

Pvalue

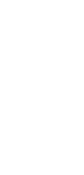


Table 2 Effect of estimates of oral herbal medicine for intrahepatic cholestasis in pregnancy in 43 included trials (Continued)

\begin{tabular}{ll}
\hline Trials & Intervention/Comparison \\
\hline Chen and Mo [55], 2005 & $\begin{array}{l}\text { Self prescribed prescription plus DXM and VK3 versus DXM and VK3 } \\
\text { Su et al. [60], 2015 }\end{array}$ \\
$\begin{array}{ll}\text { Lidan Decoction plus UDCA, SAMe and } \\
\text { Magnesium isoglycyrrhizinate versus UDCA, SAMe and } \\
\text { Magnesium isoglycyrrhizinate }\end{array}$ \\
Kang [61], 2014 & Kangyudan Decoction plus UDCA versus UDCA \\
Zheng [65], 2019 & Yinchen Decoction plus UDCA, SAMe and Phenobarbital versus UDCA, \\
Mao [68], 2016 & Yinchenhao Decoction plus UDCA versus UDCA \\
Liu et al. [70], 2019 & Wuling pill plus SAMe versus SAMe \\
Du and Li [72], 2014 & Yiguanjian Decoction plus UDCA and DXM versus UDCA and DXM
\end{tabular}

\section{$(95 \% \mathrm{Cl})$}

RR $0.67[0.12,3.75]$

RR $0.50[0.05,5.28]$

RR $1.00[0.07,15.38]$

RR $0.33[0.04,3.08]$

RR $0.16[0.02,1.29]$

RR $0.40[0.08,1.98]$

RR $0.38[0.16,0.92]$

RR $0.45[0.28,0.72]$

0.0009

\section{The serum ALT values}

7.1 Herbal medicine vs. Conventional medicine

Yang [32], $2015 \quad$ Yinzhihuang oral liquid vs. UDCA

Li et al. [14], $2015 \quad$ Yinchenhao Decoction vs. UDCA and SAM

$\mathrm{MD}-1.54[-2.46,-0.62]$

Huang and Liu [34], 2004 Yinchenhao Decoction vs SAMe

MD $5.70[0.50,10.90]$

Zhang et al. [35], 2006 Jiaweiyinchen Decoction vs DXM

MD $3.40[-12.37,19.17]$

$\mathrm{MD}-18.31[-46.10,9.48]$

\section{Subtotal (REM, $I^{2}=67 \%$ )}

MD $0.90[-5.10,6.90]$

7.2 Herbal medicine plus Conventional medicine versus Conventional medicine

7.2.1 Herbal medicine plus SAMe versus SAMe

Shen and Tao [43], 2009 Tuihuangguyuan Decoction

Liu et al. [46], $2013 \quad$ Yinchendanshao Decoction

$M D-11.50[-16.78,-$

Zhang [52], $2015 \quad$ Yinchenhao Decoction

6.22]

Liu et al. [70], $2019 \quad$ Wuling pill

MD $2.97[-3.73,9.67]$

Liu et al. [70], 2019 - Wuling pill

Wang et al. [71], $2015 \quad$ Yinchenhao Decoction

Zhang [73], $2005 \quad$ Dangguidihuang Decoction plus SAMe versus SAMe

7.2.2 Herbal medicine plus UDCA versus UDCA

Wang and Lai [42], 2011 Yinchenlidan Decoction

Lu [45], $2013 \quad$ Yinchenzhuyedihuang Decoction

Yin [53], $2015 \quad$ Bushenqingli Decoction

Shu [58], $2018 \quad$ Qianyinlidan Decoction

Deng [59], $2015 \quad$ Yinzhihuang oral liquid

Wei [63], $2016 \quad$ Self prescribed prescription

Gu et al. [66], $2014 \quad$ Yinchenhao Decoction

Zhu and Huang [69], Yinchenhao Decoction

$\mathrm{MD}-13.60[-19.20,-$

8.00]

$\mathrm{MD}-43.86[-47.04,-$

40.68]

$\mathrm{MD}-10.20$ [- 17.59, -

2.81]

MD -14.02 [- 19.04, - 9.00]

MD-26.10 [- 50.18, - 2.02]

MD - 72.00 [- 75.85, -

68.15]

$\mathrm{MD}-13.00[-17.50,-$

8.50]

$\mathrm{MD}-19.07[-24.70,-$

13.44]

MD - 16.98 [- 18.89, -

15.07]

$\mathrm{MD}-10.40[-16.37,-$

4.43]

$\mathrm{MD}-11.67[-17.33,-$

6.01] 2008

$\mathrm{MD}-1.37[-5.07,2.33]$

$\mathrm{MD}-8.60[-24.25,7.05]$ 
Table 2 Effect of estimates of oral herbal medicine for intrahepatic cholestasis in pregnancy in 43 included trials (Continued)

\begin{tabular}{|c|c|c|c|}
\hline Trials & Intervention/Comparison & $\begin{array}{l}\text { Effect estimates } \\
(95 \% \mathrm{Cl})\end{array}$ & Pvalue \\
\hline Wang et al. [36], 2016 & Danyu Decoction & $\begin{array}{l}\mathrm{MD}-14.85[-18.88,- \\
10.82]\end{array}$ & \\
\hline Li et al. [14], 2015 & Yinchenhao Decoction & $\begin{array}{l}\mathrm{MD}-21.00[-25.60,- \\
16.40]\end{array}$ & \\
\hline Su et al. [60], 2015 & Lidan Decoction & $\begin{array}{l}\mathrm{MD}-18.40[-23.56,- \\
13.24]\end{array}$ & \\
\hline Zheng [65], 2019 & Yinchen Decoction & $\begin{array}{l}M D-19.11[-20.98,- \\
17.24]\end{array}$ & \\
\hline Mao and He [67], 2014 & Qingganlidan Decoction & $\begin{array}{l}M D-21.10[-24.86,- \\
17.34]\end{array}$ & \\
\hline Subtotal (REM, $\left.I^{2}=34 \%\right)$ & & $\begin{array}{l}\text { MD-18.94 [- 20.91, - } \\
16.97]\end{array}$ & $<0.0001$ \\
\hline \multicolumn{4}{|c|}{ 7.2.4 Herbal medicine plus UDCA, SAMe and dexamethasone versusUDCA, SAMe and dexamethasone } \\
\hline Shan et al. [50], 2016 & Huashilidan Decoction & MD-8.69 [-13.16, - 4.22] & 0.0001 \\
\hline \multicolumn{4}{|c|}{ 7.2.5 Herbal medicine plus dexamethasone versus dexamethasone } \\
\hline Liu et al. [38], 2015 & Yinchenlidan Decoction & MD-13.33 [-17.34, - 9.32] & \\
\hline Zhao [41], 2011 & Qingyulidan Decoction & MD-10.13 [-13.93, - 6.33] & \\
\hline Zhang [54], 2008 & Self prescribed prescription & MD-19.10 [-23.90, - 14.30] & \\
\hline Chen and Mo [55], 2005 & Self prescribed prescription & MD-20.03 [-27.91, - 12.15] & \\
\hline Subtotal (REM, $\left.I^{2}=72 \%\right)$ & & $\begin{array}{l}\text { MD-15.05 [- 19.59, - } \\
10.51]\end{array}$ & $<0.0001$ \\
\hline
\end{tabular}

\section{The serum AST values}

8.1 Herbal medicine vs. Conventional medicine
Li et al. [14], 2015
Yinchenhao Decoction vs UDCA and SAM
Zhang et al. [35], 2006
Jiaweiyinchen Decoction vs DXM

\section{Overall (REM, $\left.I^{2}=72 \%\right)$}

MD $11.20[7.86,14.54]$

$\mathrm{MD}-12.90[-37.51,11.71]$

MD $2.36[-20.40,25.12] \quad 0.84$

8.2 Herbal medicine plus Conventional medicine versus Conventional medicine

8.2.1 Herbal medicine plus SAMe versus SAMe

\begin{tabular}{|c|c|}
\hline Liu et al. [46], 2013 & Yinchendanshao Decoction \\
\hline Zhang [52], 2015 & Yinchenhao Decoction \\
\hline Liu et al. [70], 2019 & Wuling pill \\
\hline Wang et al. [71], 2015 & Yinchenhao Decoction \\
\hline \multicolumn{2}{|c|}{ 8.2.2 Herbal medicine plus UDCA versus UDCA } \\
\hline Yin [53], 2015 & Bushenqingli Decoction \\
\hline Ma [56], 2010 & Self prescribed prescription \\
\hline Shu [58], 2018 & Qianyinlidan Decoction \\
\hline Wei [63], 2016 & Self prescribed prescription \\
\hline Gu et al. [66], 2014 & Yinchenhao Decoction \\
\hline
\end{tabular}

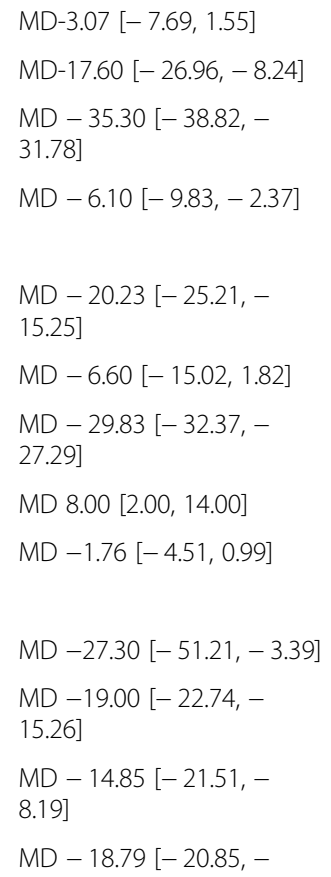


Table 2 Effect of estimates of oral herbal medicine for intrahepatic cholestasis in pregnancy in 43 included trials (Continued)

\begin{tabular}{|c|c|c|c|}
\hline Trials & Intervention/Comparison & $\begin{array}{l}\text { Effect estimates } \\
(95 \% \mathrm{Cl})\end{array}$ & Pvalue \\
\hline & & 16.73] & \\
\hline Mao and He [67], 2014 & Qingganlidan Decoction & $\mathrm{MD}-11.20[-14.27,-8.13]$ & \\
\hline \multicolumn{4}{|c|}{ 8.2.4 Herbal medicine plus UDCA, SAMe and dexamethasone versusUDCA, SAMe and dexamethasone } \\
\hline Shan et al. [50], 2016 & Huashilidan Decoction & MD-2.19 $[-6.05,1.67]$ & 0.27 \\
\hline \multicolumn{4}{|c|}{ 8.2.5 Herbal medicine plus dexamethasone versus dexamethasone } \\
\hline Liu et al. [38], 2015 & Yinchenlidan Decoction & MD-17.48 $[-24.21,-10.75]$ & \\
\hline Zhao [41], 2011 & Qingyulidan Decoction & MD-4.65 $[-8.58,-0.72]$ & \\
\hline Chen and Mo [55], 2005 & Self prescribed prescription & MD-6.54 $[-12.97,-0.11]$ & \\
\hline
\end{tabular}

\section{The average gestational age of the newborn}

9.1 Herbal medicine vs. Conventional medicine

Zhang [31], $2006 \quad$ Self prescribed prescription vs. SAMe and Reduced glutathione

MD $1.80[1.04,2.56]$

Li et al. [14], $2015 \quad$ Yinchenhao Decoction vs. UDCA and SAMe

$\mathrm{MD}-0.50[-1.31,0.31]$

Huang and Liu [34], 2004 Yinchenhao Decoction vs SAMe

MD $0.70[-0.35,1.75]$

9.2 Herbal medicine plus Conventional medicine versus Conventional medicine

Li et al. [14], $2015 \quad$ Yinchenhao Decoctionplus UDCA plus SAMe versus UDCA plus SAMe

MD $0.40[-0.37,1.17]$

Gu et al. [66], 2014 Yinchenhao Decoction plus UDCA and SAMe versus UDCA and SAMe

MD $0.46[-0.29,1.21]$

Zhu and Huang [69], Yinchenhao Decoction plus UDCA versus UDCA

MD $0.70[-0.35,1.75]$

\section{Overall (FEM, $l^{2}=0 \%$ )}

MD $0.49[0.01,0.97]$

\section{The number of premature birth}

10.1 Herbal medicine vs. Conventional medicine

Yang [32], $2015 \quad$ Yinzhihuang oral liquid vs UDCA

Zhang et al. [35], 2006 Jiaweiyinchen Decoction vs DXM

\section{Overall (FEM, $I^{2}=0 \%$ )}

10.2 Herbal medicine plus Conventional medicine versus Conventional medicine Wang et al. [36], 2016 Zhou et al. [37], 2017 Wang $X$ et al. [39], 2016 Yu [40], 2017

Zhao [41], 2011

Shen and Tao [43], 2009

Wen et al. [44], 2014

Lu [45], 2013

Tian et al. [47], 2016

Ding et al. [48], 2010

Shan et al. [50], 2016

Lan et al. [51], 2016

Zhang [52], 2015

Yin [53], 2015

Ma [56], 2010

Su et al. [60], 2015

Lidan Decoction plus UDCA, SAMe and Yinzhihuang oral liquid plus UDCA and SAMe versus UDCA and SAMe Yinchailishi Decoction plus UDCA and SAMe versus UDCA and SAMe

Danyu Decoction plus UDCA versus UDCA Yinzhijiangdansuan Decoction plus UDCA versus UDCA

Qingyulidan Decoctionplus DXM, SAMe and phenobarbital versus DXM, SAMe plus phenobarbital

Tuihuangguyuan Decoction plus SAMe versus SAMe

Self prescribed prescription plus SAMe versus SAMe

Yinchenzhuyedihuang Decoction plus UDCA versus UDCA

Kangdanyu Decoction plus Reduced glutathione, UDCA, SAMe and DXM versus Reduced glutathione, UDCA and SAMe

Qingganhuayu Decoction plus SAMe versus SAMe

RR $0.33[0.04,3.03]$

RR $0.23[0.03,1.97]$

RR $0.28[0.06,1.27]$
RR $0.27[0.08,0.90]$

RR $0.13[0.02,0.94]$

RR $0.30[0.09,1.03]$

$\operatorname{RR} 0.32[0.04,2.91]$

RR $0.45[0.15,1.42]$

RR $0.50[0.18,1.38]$

RR $0.47[0.22,1.01]$

RR $0.26[0.07,0.99]$

RR $1.50[0.84,2.68]$

RR $0.51[0.32,0.82]$

RR $0.33[0.07,1.57]$

$\operatorname{RR} 0.42[0.16,1.07]$

RR $0.29[0.10,0.79]$

RR $0.25[0.06,1.08]$

RR $0.36[0.13,1.03]$

RR $0.33[0.04,3.06]$ Magnesium isoglycyrrhizinate versus UDCA, SAMe and

Magnesium isoglycyrrhizinate

Yinchenhao Decoction plus SAMe versus SAMe

Bushenqingli Decoction plus UDCA versus UDCA

Self prescribed prescription plus UDCA versus UDCA 
Table 2 Effect of estimates of oral herbal medicine for intrahepatic cholestasis in pregnancy in 43 included trials (Continued)

\begin{tabular}{cll}
\hline Trials & Intervention/Comparison & $\begin{array}{l}\text { Effect estimates } \\
(\mathbf{9 5 \%} \mathbf{C})\end{array}$ \\
\hline Wang et al. [62], 2018 & $\begin{array}{l}\text { Yinzhihuang granule plus UDCA, SAMe and DXM versus UDCA, SAMe and } \\
\text { DXM }\end{array}$ & RR 0.18 [0.04, 0.75] \\
Wei [63], 2016 & Self prescribed prescription plus UDCA versus UDCA & RR 0.33 [0.07, 1.52] \\
Zhang [64], 2017 & Lidan Decoction plus UDCA versus UDCA & RR 0.29 [0.06, 1.32] \\
Zheng [65], 2019 & $\begin{array}{l}\text { Yinchen Decoction plus UDCA, SAMe and Phenobarbital versus UDCA, } \\
\text { SAMe and Phenobarbital }\end{array}$ & RR 0.20 [0.01, 4.04] \\
Mao and He [67], 2014 & Qingganlidan Decoction plus UDCA and SAMe versus UDCA and SAMe & RR 0.23 [0.07, 0.77] \\
Mao [68], 2016 & Yinchenhao Decoction plus UDCA versus UDCA & RR 0.49 [0.05, 5.15] \\
Liu et al. [70], 2019 & Wuling pill plus SAMe versus SAMe & RR 0.43 [0.12, 1.58] \\
Wang et al. [71], 2015 & Yinchenhao Decoction plus SAMe versus SAMe & RR 0.60 [0.17, 2.18] \\
Du and Li [72], 2014 & Yiguanjian Decoction plus UDCA and DXM versus UDCA and DXM & RR 0.31 [0.12, 0.82] \\
Overall (FEM, $\mathbf{I}^{\mathbf{2}=\mathbf{7} \%)}$ & & RR $\mathbf{0 . 4 1}[\mathbf{0 . 3 4 , 0 . 5 1 ]}$
\end{tabular}

UDCA Ursodesoxycholic acid, SAMe S-adenosylmethionine, DXM Dexamethasone, VK3 Vitamin K3, MD Mean Difference, RR Risk Ratio, FEM Fixed effect model, REM Random effect model

showed that there was no significant difference in this outcome between herbal medicine and conventional medicine (MD 0.90, 95\% CI -5.10 to $6.90, I^{2}=67 \%, P=$ $0.77,4$ trials, 300 patients) [32-35]. Two studies reported changes in maternal serum AST and the results showed that there was no significant difference in this outcome between herbal medicine and conventional medicine (MD 11.20 U/L, 95\% CI 7.86 to $14.54, P<$ 0.00001, 1 trial, 120 patients) [33, 35]. Three studies reported the gestational age of the newborn (MD and 95\% CI were 1.80 weeks, 1.04 to $2.56 ;-0.50$ weeks, -1.31 to 0.31 ; 0.70 weeks, -0.35 to 1.75 ; respectively) [31, 33, 34], meta-analysis of all the above studies could not be conducted due to the obvious statistical heterogeneity. Another trials reported the numbers of the preterm births, which showed no differences between herbal medicine and conventional medicine (RR $0.28,95 \%$ CI 0.06 to $1.27, I^{2}=0 \%, P=0.10,12$ trials, 120 patients) [32, 35].

\section{Combination of herbal medicine and conventional medicine vs. conventional medicine alone}

Thrity-nine trials contributed data to this comparison [33, 36-73].

Pruritus score Fifteen trials reported pruritus score of the pregnancy which was based on the standard Ribalta pruritus score $[37,38,41-44,46,48,51,53,59,66,70$, $71,73]$. The post-treatment score could not be synthesized due to the obvious statistical heterogeneity. Subgroup analysis showed potential good adjunctive effect of herbal medicine on decreasing the pruritus scores on the basis with SAMe (MD -0.91, 95\% CI - 1.10 to -0.72 , $I^{2}=80 \%, 7$ trials, 638 women), UDCA (MD -0.58, 95\% CI -0.79 to $-0.36, I^{2}=56 \%, 4$ trials, 273 women), combination of SAMe and dexamethasone (MD -0.69, 95\%
CI -0.80 to $-0.57, I^{2}=0 \%, 2$ trials, 276 women), and the combination of SAMe and UDCA (MD -1.68, 95\% CI -1.99 to $-1.37,1$ trial, 80 women), (MD -0.77 , 95\% CI -1.01 to -0.53 , 1 trial, 60 women).

The serum TBA value Twenty-three trials reported the serum TBA before and post-treatment [33, 36-41, 45, $46,50-55,57,60,63,65-67,71,73]$. The score could not be synthesised due to the obvious statistical heterogeneity. Subgroup analysis showed potential good adjunctive effect of herbal medicine on decreasing the serum TBA scores on the basis with UDCA (MD $3.99 \mu \mathrm{mol} / \mathrm{L}, 95 \% \mathrm{CI}-4.24$ to $-3.74, I^{2}=0 \%, 7$ trials, 522 women), SAMe (MD $-7.33 \mu \mathrm{mol} / \mathrm{L}, 95 \% \mathrm{CI}-8.50$ to $-6.15, I^{2}=0 \%, 4$ trials, 230 women), dexamethasone (MD $-23.30 \mu \mathrm{mol} / \mathrm{L}, 95 \% \mathrm{CI}-24.61$ to $-21.99,1$ trial, 86 women), combination of UDCA and SAMe (MD $7.62 \mu \mathrm{mol} / \mathrm{L}, 95 \% \mathrm{CI}-12.97$ to $-2.27, I^{2}=98 \%, 7$ trials, 652 women), and the combination of UDCA and SAMe and dexamethasone (MD $-3.54 \mu \mathrm{mol} / \mathrm{L}, 95 \% \mathrm{CI}-4.03$ to $-3.05, I^{2}=8 \%, 4$ trials, 417 women).

The number of adverse birth events Six trials reported no stillbirths or neonatal deaths post-treatment $[53,55$, $61,63,69,71]$. One trial reported one neonatal deaths post-treatment in conventional medicine group [60].

Twenty-four trials reported the number of fetal distress $[36-38,40,41,43,45,47,49-51,53,60,62-65$, 67-73], 18 trials reported the number of asphyxia neonatorum [37, 39-41, 43, 45, 47-53, 56, 61, 65, 67, 71], 22 trials reported the number of cesarean section [33, $36-41,43,45,47,48,53,55,56,61,63,64,67,69,70$, $72,73]$, and another 14 trials reported the number of postpartum hemorrhage [39-41, 43, 47, 50, 53, 55, 60, $61,65,68,70,72]$. Significantly lower incidence of fetal 
distress (RR $0.41,95 \%$ CI 0.32 to $0.51, I^{2}=0 \%, 24$ trials, 1915 women), asphyxia neonatorum (RR 0.35 , 95\%CI 0.25 to $0.49, I^{2}=0 \%, 18$ trials, 1492 women), cesarean section (RR 0.73, 95\% CI 0.63 to $0.85, I^{2}=65 \%, 22$ trials, 1974 women), postpartum hemorrhage (RR $0.45,95 \%$ CI 0.28 to $0.72, I^{2}=0 \%, 14$ trials, 1130 women) were observed in the combination group compared to the conventional medicine group.

Liver function blood test results Twenty-five trials reported the serum ALT values [33, 36, 38, 39, 41-43, 45, $46,50,52-55,58-60,63,65-67,69-71,73]$. The data could not be synthesized due to the obvious statistical heterogeneity. Results from single study or subgroup meta-analysis showed potential good adjunctive effect of herbal medicine on decreasing the serum ALT values on the basis of UDCA (MD -19.91 U/L, 95\% CI -35.42 to $-4.39, I^{2}=99 \%$, 9 trials, 625 women), dexamethasone (MD $-15.05 \mathrm{U} / \mathrm{L}, 95 \% \mathrm{CI}-19.59$ to $-10.51, I^{2}=72 \%, 4$ trials, 432 women), and the combination of UDCA and SAMe (MD -18.94 U/L, 95\% CI -20.91 to $-16.97, I^{2}=$ $34 \%, 5$ trials, 506 women), the combination of UDCA, SAMe and dexamethasone (MD -8.69 U/L, 95\% CI 13.16 to -4.22 , 1 trial, 96 women). Six trials reported there is no statistical difference between the combination treatment and SAMe alone in post-treatment serum ALT value (MD $-15.14 \mathrm{U} / \mathrm{L}, 95 \% \mathrm{CI}-30.79$ to $0.50, I^{2}=98 \%, 6$ trials, 410 women). Detail of the results were shown in Table 2.

Eighteen trials reported the serum AST values [33, 36, $38,41,46,50,52,53,55,56,58,60,63,65-67,70,71]$. Results from single study or subgroup meta-analysis showed potential good adjunctive effect of herbal medicine on decreasing the serum AST values on the basis with the combination of UDCA and SAMe (MD -16.38 $\mathrm{U} / \mathrm{L}, 95 \% \mathrm{CI}-20.49$ to $-12.27, I^{2}=79 \%, 5$ trials, 476 women), dexamethasone (MD -9.23 U/L, 95\% CI - 16.71 to $-1.76, I^{2}=81 \%, 3$ trials, 346 women). There is no statistical difference of herbal medicine on decreasing the serum AST values on the basis with UDCA, SAMe, and the combination of UDCA and SAMe and dexamethasone. Detail of the results were also shown in Table 2.

The average gestational age of the newborn One trial reported the average gestational age of the newborn which indicated that there was no difference between thecombination group and conventional medicine group (MD 0.49 weeks, 95\% CI 0.01 to $0.97,3$ trials, 263 women) $[33,66,69]$.

The number of premature birth Twenty-six trials reported the number of premature birth [32, 36, 37, 39$41,43-45,47,48,50-53,60,62-65,67,68,70-72]$.
Significantly lower incidence of Premature birth was observed in the combination group than thecontrol group (RR 0.41, 95\% CI 0.34 to $0.51, I^{2}=7 \%, 26$ trials, 2199 women).

\section{Adverse events}

Five trials reported the number of adverse events [48, 53, $55,68,70]$. One trial reported six cases of nausea, headache and flushin in treatment group [48]. One trial reported one case of loose stool, disappeared in the following course, two cases of nausea [53]. One trial reported no adverse events during the treatment [55]. One trial reported one case of headache and one case of tachycardia in treatment group, and one case of tachycardia and one case of constipation in control group [68]. Another trial reported two cases of nausea, two cases of acid reflux, one case of bloating in treatment group, and one case of acid reflux, two cases of bloating, one case of nausea, one case of rash in control group [70].

\section{Funnel plot}

A funnel plot analysis of 24 trials was performed to examine outcome for the serum TBA value of patients. The result showed potential asymmetry (Fig. 3). The potential asymmetry may be caused by small study effects, different methodological quality, or even Heterogeneity in intervention effects.

\section{Trial sequential analysis (TSA)}

We conducted TSA with the data from the two metaanalyses above. For the outcome of numbers of fetal distress in comparing herbal medicine combined conventional medicine to conventional medicine alone, TSA illustrated that the cumulative Z-curve across the traditional boundary of $5 \%$ significance (horizontal line) as well as the monitoring boundaries (inward sloping curves) (see Fig. 4). After the sixth study, the significance testing had been performed each time a new trial was added to the meta-analysis, which means the sample size achieved the required 349 participants and we had enough power to confirm the evidence (that with adjunction of herbal medicine, the therapy may decrease $12 \%$ more fetal distress) controlling for the risk of random error.

For the outcome of numbers of asphyxia neonatorum in the same comparison, the result was similar with the two crossings. TSA also illustrated that the cumulative Z-curve across the horizontal line and the inward sloping curves (see Fig. 5), which means the sample size achieved the required 376 participants and we had enough power to confirm the evidence (that the combination therapy may decrease $10 \%$ more cases of asphyxia neonatorum). 


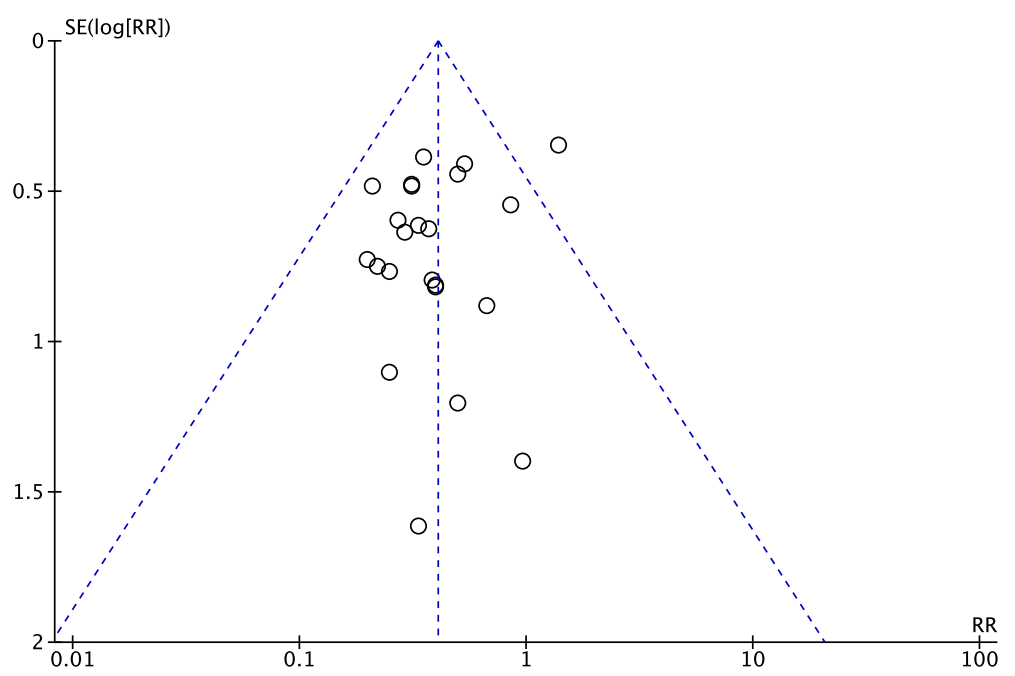

Fig. 3 Funnel plot assessing outcomes of the number of fetal distress reported in 24 randomized controlled trials

\section{Discussion}

\section{Summary of main findings}

Totally 43 trials with 3556 women were included, all of them were assessed as having high risk of bias. Results from these trials showed potential better effect of herbal medicine when combined with conventional medicine on decreasing pruritus scores, reducing adverse birth events (which was supported by the TSA results) and improving the liver functions according to the serum
TBA, ALT, AST values compared to conventional medicine alone. However, due to the poor methodology of the included studies and the obvious statistical heterogeneity among trials, quality of the evidence for all these outcomes were "low" and "very low" according to the GRADE assessment (see Tables 3 and 4). When compared to conventional medicine, the single application of herbal medicine showed no better/worse effect for the condition, we could not draw any firm conclusion on

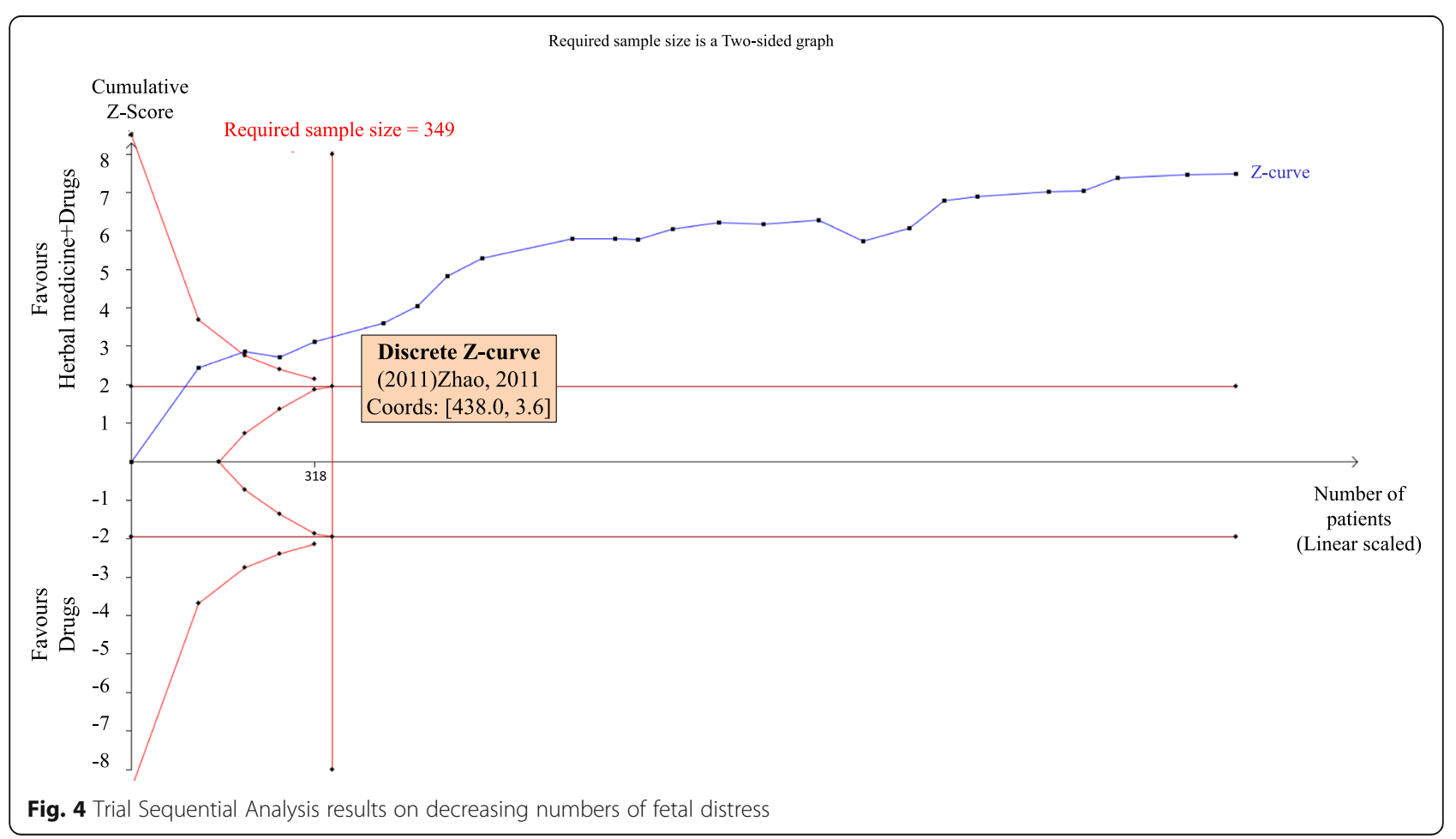


Required sample size is a Two-sided graph

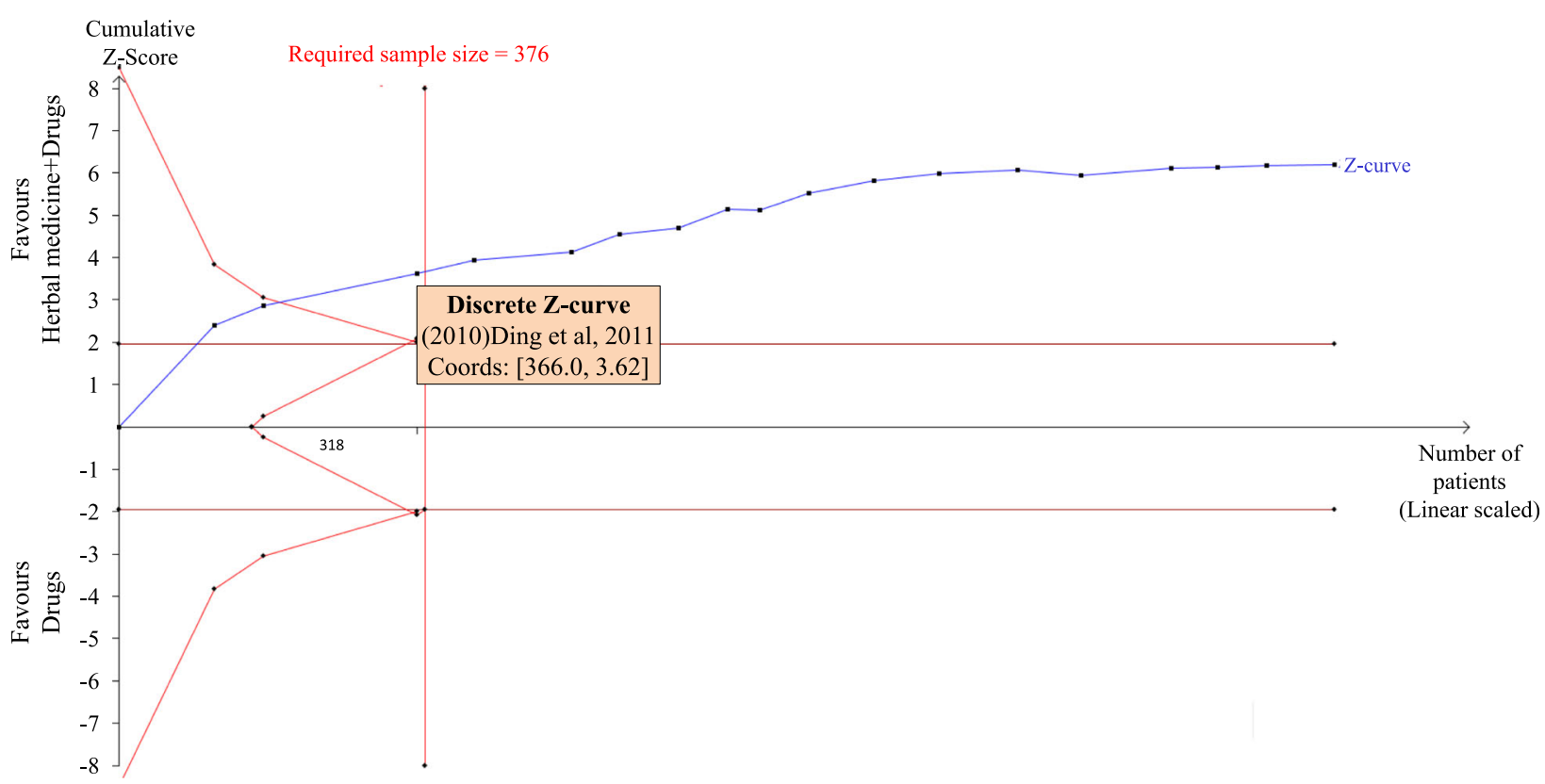

Fig. 5 Trial Sequential Analysis results on decreasing numbers of asphyxia neonatorum

this type of comparison due to the small sample size of involved trials. Furthermore, few data were reported to indicate the adverse events in herbal medicine group, which also made the safety of the herbal medicine was unclear.

\section{Compare to the previous studies}

The results of a meta-analysis preliminarily showed that traditional Chinese medicine combined with conventional medicine (or physical therapy) had a certain therapeutic effect on neonatal jaundice in treatment of recurrent spontaneous abortion [74]. Another metaanalysis showed that Yinzhihuang Oral Liquid was more effective in improving itching symptoms of pregnant women, reducing serum total bilirubin, total bilirubin, glycocholic acid and increasing neonatal weight of newborns than the control group in treatment of intrahepatic cholestasis of pregnancy [75]. The third meta-analysis showed that Yinchenhao Decoction had better effect on treating neonatal jaundice, shortening recovery time of serum total bilirubin and jaundice subsidence time than that of conventional medicine alone [76]. All these findings indicated that herbal medicine may have effect on improving the liver function of pregnancy women, which was consistent with our findings.

Since we did not find any other review focused on this disease through the literature searching, this is the probably the first study assessed the herbal medicine for ICP and the current evidence may support the effectiveness of combination of herbal medicine and conventional medicine for decreasing the maternal pruritus scores, the serum TBA, and the number of fetal distress, asphyxial events or asphyxia neonatorum events related to this condition.

\section{Implications for practice}

Though we only got "very low" quality evidence to support the adjunctive effect of herbal medicine in treating ICP. The results showed a statistically meaningful advantage of herbal medicine on improving the symptoms or reducing adverse birth events when combined with conventional medicine. Considering the uncertain safety of the herbal product, we suggest the application of herbal medicine would be recommended in consideration of the expertise and experience of the clinician. Treatment duration would be 10 to 14 days according to this review. The most frequently used prescription in our review is Yinchenhao Decoction, in which Yinchen and Zhizi are the core component. Bian found that Yinchenhao decotion can induce liver fibrosis by dimethylnitrosamine, reduce hydroxyproline and improve liver function and hepatic histology after 2 weeks of treatment in rats [77]. Mentimes, a systematic review indicated that Yinchenhao decotion can significantly improve 
Table 3 Summary of finding table of herbal medicine compared to conventional medicine for women with intrahepatic cholestasis in pregnancy

\begin{tabular}{|c|c|c|c|c|c|c|}
\hline \multicolumn{7}{|c|}{$\begin{array}{l}\text { Patient or population: Women with intrahepatic cholestasis in pregnancy } \\
\text { Settings: Outpatient department/ Inpatient department } \\
\text { Intervention: Herbal medicine } \\
\text { Comparison: Conventional medicine }\end{array}$} \\
\hline \multirow[t]{3}{*}{ Outcomes } & \multicolumn{2}{|c|}{ Illustrative comparative risks ${ }^{\mathrm{a}}(95 \% \mathrm{Cl})$} & \multirow{3}{*}{$\begin{array}{l}\text { Relative } \\
\text { effect } \\
(95 \% \mathrm{Cl})\end{array}$} & \multirow{3}{*}{$\begin{array}{l}\text { No of } \\
\text { Participants } \\
\text { (studies) }\end{array}$} & \multirow{3}{*}{$\begin{array}{l}\text { Quality of } \\
\text { the } \\
\text { evidence } \\
\text { (GRADE) }\end{array}$} & \multirow[t]{3}{*}{ Comments } \\
\hline & Assumed risk & Corresponding risk & & & & \\
\hline & $\begin{array}{l}\text { Conventional } \\
\text { medicine }\end{array}$ & herbal medicine & & & & \\
\hline $\begin{array}{l}\text { Ribalta pruritus } \\
\text { score } \\
\text { Scale from: } 0 \text { to } 4 .\end{array}$ & & $\begin{array}{l}\text { The mean Ribalta pruritus score in the intervention } \\
\text { groups was } \mathbf{0 . 6 8} \text { lower ( } 0.88 \text { to } 0.47 \text { lower) }\end{array}$ & & $\begin{array}{l}120 \\
\text { (2 studies) }\end{array}$ & $\begin{array}{l}\oplus \ominus \Theta \Theta \\
\text { very low } \text { low }^{\mathrm{b}, \mathrm{c}}\end{array}$ & \\
\hline $\begin{array}{l}\text { The serum TBA } \\
\text { value }\end{array}$ & & $\begin{array}{l}\text { The mean the serum TBA value in the intervention } \\
\text { groups was } \mathbf{1 . 6 7} \text { lower ( } 7.69 \text { lower to } 4.35 \text { higher) }\end{array}$ & & $\begin{array}{l}180 \\
\text { (2 studies) }\end{array}$ & $\begin{array}{l}\oplus \ominus \Theta \Theta \\
\text { very low } \text { low,c,d }^{\mathrm{b}}\end{array}$ & \\
\hline $\begin{array}{l}\text { The number of fetal } \\
\text { distress }\end{array}$ & 192 per 1000 & $\begin{array}{l}148 \text { per } 1000 \\
\text { (83 to } 267)\end{array}$ & $\begin{array}{l}\text { RR } 0.77 \\
(0.43 \text { to } \\
1.39)\end{array}$ & $\begin{array}{l}218 \\
\text { (3 studies) }\end{array}$ & $\underset{\text { low }^{b}}{\oplus \oplus} \oplus \ominus$ & \\
\hline $\begin{array}{l}\text { The number of } \\
\text { asphyxia } \\
\text { neonatorum }\end{array}$ & 33 per 1000 & $\begin{array}{l}33 \text { per } 1000 \\
(2 \text { to } 559)\end{array}$ & $\begin{array}{l}\text { RR } 1 \\
\text { (0.06 to } \\
16.76)\end{array}$ & $\begin{array}{l}60 \\
\text { (1 study) }\end{array}$ & $\begin{array}{l}\oplus \oplus \Theta \Theta \\
\text { low }^{c e}\end{array}$ & \\
\hline
\end{tabular}

GRADE Working Group grades of evidence

High quality: Further research is very unlikely to change our confidence in the estimate of effect

Moderate quality: Further research is likely to have an important impact on our confidence in the estimate of effect and may change the estimate

Low quality: Further research is very likely to have an important impact on our confidence in the estimate of effect and is likely to change the estimate

Very low quality: We are very uncertain about the estimate

$C /$ Confidence interval, $R R$ Risk ratio

${ }^{\text {a }}$ The basis for the assumed risk (e.g. the median control group risk across studies) is provided in footnotes. The corresponding risk (and its $95 \%$ confidence

interval) is based on the assumed risk in the comparison group and the relative effect of the intervention (and its $95 \% \mathrm{Cl}$ )

${ }^{\mathrm{b}}$ There were very serious limitations of methodological quality of included trials according to the risk of bias assessment

'Too small sample size

${ }^{\mathrm{d}}$ There were very serious statistical heterogeneity among included trials

${ }^{\mathrm{e}}$ There were serious limitations of methodological quality of included trials according to the risk of bias assessment

cholestasis by reducing elevated serum markers [78]. Practitioners may choose to use modified Yinchenhao Decoction in treating this condition.

\section{Implications for future studies}

There are some deficiencies in this study that should be improved in future studies. In terms of scheme design and method application, most studies did not give a clear introduction to the method of randomization and blinding. Although the blind method may not be easy to operate for patients due to the difficulty in the operation of herbal medicine placebo, a blind method should be implemented for the outcome assessors or statisticians to avoid bias. Secondly, in terms of the data analysis, missing data should be handled with appropriate statistical methods. Reporting of the trial should follow the standard of CONSORT. Besides, five databases have been searched, but no studies that are published in English can be included, which suggests that relevant studies are possibly published mainly in Chinese. Since the access to databases such as Allied and Complementary Medicine (AMED) and the Cumulative Index to Nursing and Allied Health Literature (CINAHL) is not available for searching within the region of our researchers, there might be the possibility to miss studies. More English databases should be considered to be searched in the future.

High quality studies are needed to verify the adjuvant efficacy and safety of Chinese herbal medicine. Meanwhile, cost effect analysis may be considered to be conducted in the future.

\section{Conclusions}

This review found "very low" quality evidence which support the effectiveness of combination of herbal medicine and conventional medicine for decreasing the maternal pruritus scores, the serum TBA, and the number of adverse birth events. TSA analysis showed the results of benefit of combination of herbal medicine and conventional medicine for decreasing the number of fetal 
Table 4 Summary of finding table of combination of herbal medicine and conventional medicine compared to conventional medicine for women with intrahepatic cholestasis in pregnancy

\begin{tabular}{|c|c|c|c|c|c|c|}
\hline \multicolumn{7}{|c|}{$\begin{array}{l}\text { Combination of herbal medicine and conventional medicine compared } \\
\text { in pregnancy } \\
\text { Patient or population: Women with intrahepatic cholestasis in pregnancy } \\
\text { Settings: } \\
\text { Intervention: Combination of herbal medicine and conventional medicine } \\
\text { Comparison: Conventional medicine }\end{array}$} \\
\hline \multirow[t]{3}{*}{ Outcomes } & \multicolumn{2}{|c|}{ Illustrative comparative risks ${ }^{\mathrm{a}}(95 \% \mathrm{Cl})$} & \multirow{3}{*}{$\begin{array}{l}\text { Relative } \\
\text { effect } \\
(95 \% \mathrm{Cl})\end{array}$} & \multirow{3}{*}{$\begin{array}{l}\text { No of } \\
\text { Participants } \\
\text { (studies) }\end{array}$} & \multirow{3}{*}{$\begin{array}{l}\text { Quality of } \\
\text { the evidence } \\
\text { (GRADE) }\end{array}$} & \multirow[t]{3}{*}{ Comments } \\
\hline & Assumed risk & Corresponding risk & & & & \\
\hline & $\begin{array}{l}\text { Conventional } \\
\text { medicine }\end{array}$ & $\begin{array}{l}\text { Combination of herbal medicine and } \\
\text { conventional medicine }\end{array}$ & & & & \\
\hline $\begin{array}{l}\text { Ribalta pruritus } \\
\text { score } \\
\text { Scale from: } 0 \text { to } 4 .\end{array}$ & & $\begin{array}{l}\text { The mean Ribalta pruritus score in the intervention } \\
\text { groups was } \mathbf{0 . 8 3} \text { lower ( } 0.98 \text { to } 0.67 \text { lower) }\end{array}$ & & $\begin{array}{l}1327 \\
\text { (15 studies) }\end{array}$ & $\begin{array}{l}\oplus \Theta \Theta \Theta \\
\text { very low } \mathbf{l}^{\mathrm{b}, c}\end{array}$ & \\
\hline $\begin{array}{l}\text { The serum TBA } \\
\text { value }\end{array}$ & & $\begin{array}{l}\text { The mean the serum TBA value in the intervention } \\
\text { groups was } \mathbf{7 . 6 2} \text { lower ( } 12.97 \text { to } 2.27 \text { lower) }\end{array}$ & & $\begin{array}{l}652 \\
\text { (23 studies) }\end{array}$ & $\begin{array}{l}\oplus \ominus \Theta \Theta \\
\text { very low } \text { lo }^{\mathrm{b}, \mathrm{c}}\end{array}$ & \\
\hline $\begin{array}{l}\text { The number of fetal } \\
\text { distress }\end{array}$ & 207 per 1000 & $\begin{array}{l}85 \text { per } 1000 \\
(66 \text { to } 106)\end{array}$ & $\begin{array}{l}\text { RR } 0.41 \\
(0.32 \text { to } \\
0.51)\end{array}$ & $\begin{array}{l}1915 \\
\text { (24 studies) }\end{array}$ & $\underset{\text { low }^{b}}{\oplus \oplus} \oplus \ominus$ & \\
\hline $\begin{array}{l}\text { The number of } \\
\text { asphyxia } \\
\text { neonatorum }\end{array}$ & 155 per 1000 & $\begin{array}{l}\mathbf{5 4} \text { per } \mathbf{1 0 0 0} \\
(39 \text { to } 76)\end{array}$ & $\begin{array}{l}\text { RR } 0.35 \\
(0.25 \text { to } \\
0.49)\end{array}$ & $\begin{array}{l}1492 \\
\text { (18 studies) }\end{array}$ & $\underset{\text { low }^{b}}{\oplus \oplus} \oplus \ominus$ & \\
\hline
\end{tabular}

GRADE Working Group grades of evidence

High quality: Further research is very unlikely to change our confidence in the estimate of effect

Moderate quality: Further research is likely to have an important impact on our confidence in the estimate of effect and may change the estimate Low quality: Further research is very likely to have an important impact on our confidence in the estimate of effect and is likely to change the estimate Very low quality: We are very uncertain about the estimate

$\mathrm{Cl}$ Confidence interval, $R R$ Risk ratio

${ }^{a}$ The basis for the assumed risk (e.g. the median control group risk across studies) is provided in footnotes. The corresponding risk (and its $95 \%$ confidence interval) is based on the assumed risk in the comparison group and the relative effect of the intervention (and its $95 \% \mathrm{Cl}$ )

${ }^{b}$ There were very serious limitations of methodological quality of included trials according to the risk of bias assessment

'There were very serious statistical heterogeneity among included trials

distress and asphyxia neonatorum had enough statistical power. More high-quality trials are still needed to prove the superior effect and safety of herbal medicine as adjunctive treatment for this disease.

\section{Supplementary information}

Supplementary information accompanies this paper at https://doi.org/10. 1186/s12906-020-03097-x.

Additional file 1. Supplementary Material 1. The ingredients of included herbal patents.

\section{Acknowledgements}

Not applicable.

\section{Authors' contributions}

PRY, NMD and YZY registered protocol and analyzed basic characteristics of trials. WRT and CN analyzed data, and were major contributors in writing the manuscript. CHJ ensure that original data, original figures, materials and code upon which the submission is based are preserved following best practices in the field so that they are retrievable for reanalysis; and confirm that data, figures, materials and code presentation accurately reflects the original; and foresee and minimize obstacles to the sharing of data, materials, code described in the work. CHJ also ensure that the entire author group is fully aware of and in compliance with best practices in the discipline of publication. All authors read and approved the final manuscript.

\section{Funding}

Hui-Juan Cao is supported by the National Natural Science Foundation of China (81804000).

Availability of data and materials

All data generated or analysed during this study are included in this published article and its supplementary information files.

Ethics approval and consent to participate Not applicable.

Consent for publication

Not applicable.

Competing interests

The authors declare that they have no competing interests.

Author details

'Dongzhimen Hospital, Beijing University of Chinese Medicine, Beijing, China. ${ }^{2}$ Beijing University of Chinese Medicine, Beijing, China. ${ }^{3}$ Centre for Evidence Based Chinese Medicine, Beijing University of Chinese Medicine, Beijing, China.

Received: 6 April 2020 Accepted: 28 September 2020

Published online: 07 October 2020

References

1. Geenes V, Williamson C. Intrahepatic cholestasis of pregnancy. World J Gastroenterol. 2009;15(17):2049-66. https://doi.org/10.3748/wjg.15.2049. 
2. Manzotti C, Casazza G, Stimac T, Nikolova D, Gluud C. Total serum bile acids or serum bile acid profile, or both, for the diagnosis of intrahepatic cholestasis of pregnancy. Cochrane Database Syst Rev. 2017;7:CD012546.

3. Mullenbach R, Bennett A, Tetlow N, et al. ATP8B1 mutations in British cases with intrahepatic cholestasis of pregnancy. Gut. 2005;54:829-34 PMID: 15888793.

4. Fagan EA. Intrahepatic cholestasis of pregnancy. Clin Liver Dis. 1999;3:603-32.

5. Tran TT, Ahn J, Reau NS. ACG Clinical Guideline: Liver Disease and Pregnancy. Am J Gastroenterol; Cambridge. 2016;111(2):176-94. https://doi. org/10.1038/ajg.2015.430.

6. Fisk NM, Bye WB, Storey GN. Maternal features of obstetric cholestasis: 20 years experience at King George V hospital. Aust N Z J Obstet Gynaecol. 1988;28:172-6.

7. Mays JK. The active management of intrahepatic cholestasis of pregnancy. Curr Opin Obstet Gynecol. 2010;22:100-3.

8. Royal College of Obstetricians and Gynaecologists: RCOG Green-top Guideline no.43. Obstetric Cholestasis; 2011. p. 04.

9. Arrese $\mathrm{M}, \mathrm{Macias} \mathrm{Rl}, \mathrm{Briz} \mathrm{O}$, et al. Molecular pathogenesis of intrahepatic cholestasis of pregnancy. Expert Rev Mol Med. 2008;10:e9.

10. Dixon PH, van Mil SW, Chambers J, et al. Contribution of variant alleles of ABCB11to susceptibility to intrahepatic cholestasis of pregnancy. Gut. 2009; 58:537-44.

11. Floreani A, Caroli D, Lazzari R, et al. Intrahepatic chole-stasis of pregnancy: new insights into its pathogenesis [J]. Matern Fetal Neonatal Med. 2013; 26(14):1410-5.

12. Reyes H, Báez ME, González MC, et al. Selenium, zinc and copper plasma levels in intrahepatic cholestasis of pregnancy, in normal pregnancies and in healthy individuals in Chile. J Hepatol. 2000;32:542-9.

13. Heinonen S, Kirkinen P. Pregnancy outcome with intrahepatic cholestasis. Obstet Gynecol. 1999;94(2):189-93.

14. Salame AA, Jaffal MJ, Mouanness MA, Nasser Eddin AR, Ghulmiyyah LM. Unexplained first trimester intrahepatic cholestasis of pregnancy: a case report and literature review. Case Rep Obstet Gynecol. 2019;2019:4980610.

15. Gonzalez MC, Reyes H, Arrese M, Figueroa D, Lorca B, Andresen M, Segovia $\mathrm{N}$, Molina C, Arce S. Intrahepatic cholestasis of pregnancy in twin pregnancies. J Hepatol. 1989;9(1):84-90.

16. Celik S, Caliskan C. The impact of assisted reproductive Technology in Twin Pregnancies Complicated by intrahepatic Cholestasis of pregnancy: a retrospective cohort study. Z Geburtshilfe Neonatol. 2020. https://doi.org/10. 1055/a-1129-7358.

17. Brites $D$, Rodrigues CMP, Van-Zeller $H$, Brito A, Silva R. Relevance of serum bile acid profile in the diagnosis of intrahepatic cholestasis of pregnancy in an high incidence area: Portugal. Eur J Obstet Gynecol Reprod Biol. 1998;80: 31-8.

18. Glantz A, Marschall HU, Mattsson LA. Intrahepatic cholestasis of pregnancy: relationships between bile acid levels and fetal complication rates. Hepatology. 2004;40(2):467-74.

19. Diken Z, Usta IM, Nassar AH. A clinical approach to intrahepatic cholestasis of pregnancy. Am J Perinatol. 2014;31:1-8.

20. Ozkan S, Ceylan Y, Ozkan OV, Yildirim S. Review of a challenging clinical issue: intrahepatic cholestasis of pregnancy. World J Gastroenterol. 2015; 21(23):7134-41.

21. Gurung V, Middleton P, Milan SJ, Hague W, Thornton JG. Interventions for treating cholestasis in pregnancy. Cochrane Database Syst Rev. 2013;2013(6): CD000493.

22. Bacq $Y$, Sentilhes $L$, Reyes $H B$, et al. Efficacy of ursodeoxycholic acid in treating intrahepatic cholestasis of pregnancy: a meta-analysis. Gastroenterology. 2012;143(6):1492-501.

23. Ding $Y$, Zhao L, Huang ZH, Mei H, Dong HF, Dong JH. Therapeutic effects of emodin on acute cholestatic hepatitis and mechanism thereof: experiment with rats. Nat Med J China. 2009:89(10):699-703.

24. Ding $Y, X U F$, Xiong $X L$, Li HR. Effect of emodin on expression of farnesoid $X$ receptor in rats with acute cholestatic hepatitis. Chin J Contemp Pediatr. 2014;16(4):424-9.

25. Zhou F, Xu H. Effect of emodin on P-gp expression in intrahepatic cholestatic rats. China J Chin Meteria Medica. 2010;35(7):908-11.

26. Ren JY, Wang LT, Lei CD. Comparative trial on efficacy of qinggan granule and bushen granule in treating chronic hepatitis C. Chin J Integr Tradit Med. 2001;21:645-8.

27. Zhang B, Wan MB, Wang LT. Therapeutic effect of Bushen Rougan recipe on hepatic fibrosis in rats. J Chin Integr Med. 2005;3(2):132-5.
28. Wu T, Chang MJ, Xu YJ, Li XP, Du G, Liu D. Protective effect of Calculus Bovis Sativus on intrahepatic cholestasis in rats induced by anaphthylisothiocyanate. Am J Chin Med. 2013;41(6):1393-405.

29. Chen KL, Bi KS, Han F, et al. Evaluation of the protective effect of Zhi-Zi-daHuang decoction on acute liver injury with cholestasis induced by anaphthylisothiocyanate in rats. J Ethnopharmacol. 2015;172:402-9.

30. Lian YX, Long XY, Wu WF. Clinical and experimental trial of shuangcao tuihuang granule-1 in treating severe jaundice of acute icterohepatitis. Chin J Integr Tradit Med. 2001;21(9):649-51.

31. Zhang LX. Clinical observation on traditional Chinese medicine treatment of intrahepatic cholestasis of pregnancy. Liaoning J Tradit Chin Med. 2006;12: 1609.

32. Yang WX. To observe the curative effect of Yinzhihuang oral liquid on liver and gallbladder damp heat in intrahepatic cholestasis of pregnancy. Guangzhou Univ Tradit Chin Med. 2015.

33. Li JW, Niu JM, Rao ML, Zhou P, Zhen YH, Cai RY. Effect of integrated Chinese and medicine in treating intrahepatic Cholestasis of pregnancy. J New Chin Med. 2015;47(09):95-7.

34. Huang JY, Liu H. Analysis on therapeutic effect of Western and Chinese drug in treating intrahepatic Cholestasis pregnancy. Chinese J Integr Tradit Med. 2004;04:309-11.

35. Zhang X, Wang P, Zhang L. Clinical observation of Jiawei Yinchen decoction in treating intrahepatic cholestasis during pregnancy. Liaoning J Tradit Chin Med. 2006;12:1590-1.

36. Wang XY, Qiu MJ, Lu T, Chen YJ. Curative effect observation of Yinzhihuang combined with ursodesoxycholic acid and transmetil in treating intrahepatic cholestasis during gestation period. J Nanjing Univ Tradit Chin Med. 2016;32(06):595-7.

37. Zhou XM, Wu RX, Zhao XQ, et al. Clinical observation of Yin chai Lishi decoction combined with medicine in the treatment of intrahepatic cholestasis of pregnancy. Nei Mongol J Tradit Chin Med. 2017;36(02):89-90.

38. Liu LZ, Wang HM, Ye JH. Effect of adenosylmethionine combined with Yin Chen and Lidan fang on intrahepatic cholestasis of pregnancy. J Reprod Med. 2015;24(04):328-30.

39. Wang X, Bao YL, Yu XM. Clinical observation of intrahepatic cholestasis of pregnancy with medicine combined with Danyufang. Hebei Med. 2016; 22(01):49-52.

40. Yu H. Clinical study on intrahepatic cholestasis of pregnancy by Yin Zhi Jiang Dan Suan prescription combined with UDCA therapy. Guangxi University of Chinese Medicine; 2017.

41. Zhao RF. Clinical analysis of treatment of intrahepatic cholestasis during pregnancy by integrated traditional Chinese and medicine. Modern J Integr Tradit Chin Med. 2011;20(35):4497-8.

42. Wang JD, Lai QM. Thrity-five cases of intrahepatic cholestasis during pregnancy by integrated traditional Chinese and medicine. Zhejiang J Integr Tradit Chin Med. 2011;21(02):102-3.

43. Shen $\mathrm{CH}$, Tao Y. Thirty cases of intrahepatic cholestasis during pregnancy by integrated traditional Chinese and medicine. Zhejiang J Tradit Chin Med. 2009:44(05):347-8

44. Wen YL, Zhang ZW, Zhang XW. Clinical analysis of treatment of intrahepatic cholestasis during pregnancy by integrated traditional Chinese and medicine. Asia-Pacific Tradit Med. 2014;10(13):87-8.

45. Lv BZ. Clinical observation on treatment of ICP by Yin Chen Zhu Ye Di Huang prescription combined with UDCA therapy. Modern J Integr Tradit Chin Med. 2013:22(14):1514-6.

46. Liu F, Lin YF, Jiang LP, Len LZ. Clinical observation about treatment on intrahepatic Cholestasis of Pregnancyin mid trimester of pregnancy with Yinchen Danshao decoction. J Liaoning Univ Tradit Chin Med. 2013;15(10):104-6.

47. Tian H, Lei YJ, Ge J. Clinical effect of Ursodeoxycholic acid combined with Kangdanyu decoction on intrahepatic Cholestasis of pregnancy and its influence on pregnancy outcome. Guiding J Tradit Chin Med Pharm. 2016; 22(05):86-9.

48. Liu LZ, Wang HM, Ye JH. Effect of Adenosylmethionine combined with Yinchen Lidan decoction on intrahepatic Cholestasis of pregnancy. J Reprod Med. 2015;24(04):328-30

49. Zhang XL, Xiao HQ, Chen XY, Zhang CL. Clinical study on Qingdanzhiyang decoction in the treatment of intrahepatic Cholestasis of pregnancy. J Guangzhou Univ Tradit Chin Med. 2014;31(01):24-7.

50. Shan TF, Wang P, Zhang Y. Clinical observation on 48 cases of intrahepatic Cholestasis of pregnancy treated by Huashilidan decoction combined with medicine. Chin J Tradit Med Sci Technol. 2016;23(06):706-7. 
51. Lan XL, Rebiyemu A, Sun KX. Therapeutic effect of integrated traditional Chinese and medicine on intrahepatic cholestasis of pregnancy. Zhejiang Clin Med J. 2016;3:484-5.

52. Zhang ZQ. Clinical observation on yinchenhao decoction combination with ademetionine in the treatment of intrahepatic cholestasis of pregnancy. China Continuing Med Educ. 2015;7(30):193-4

53. Yin GZ. Clinical study on intrahepatic cholestasis of pregnancy by kindneytonifying and clearing joint ursodesoxycholic acid therapy. Nanjing Univ Tradit Chin Med. 2015

54. Zhang YW. Therapeutic effect of integrated traditional Chinese and medicine on intrahepatic cholestasis of pregnancy. J Med Theory Pract. 2008;10:1198-200.

55. Chen XY, Mo FJ. The combination of traditional Chinese medicine and dexamethasone in the treatment of intrahepatic cholestasis of pregnancy. Sichuan Med J. 2005:06:659-60.

56. Ma ZF. Treatment of 35 cases of intrahepatic cholestasis of pregnancy by integrated traditional Chinese and medicine. Chin J Ethnomed Ethnopharm. 2010;19(19):113-4.

57. Zhang $H$, Tan XY, Wu LL, Xie YT. Analysis of the effect of integrated traditional Chinese and medicine therapy in treatment of intrahepatic cholestasis of pregnancy. China Med Pharm. 2016;6(17):56-8.

58. Shu XF. Forty eight cases treating intrahepatic cholestasis of pregnancy with Qianyinlidan decoction and Ursodesoxycholic acid tablet. Chin J Tradit Med Sci Technol. 2018;25(02):247-8.

59. Deng CX. Wormwood Zhi yellow oral liquid combined with medicine in the treatment of intrahepatic cholestasis during pregnancy 60 cases observed. J Front Med. 2015;5(1):35-6. https://doi.org/10.3969/j.issn.2095-1752.2015.01. 017.

60. Su WH, Zhang YP, Luo QB. Combination of "Lidan decoction" and medicine for intrahepatic cholestasis in pregnant women. Shanghai J Tradit Chin Med. 2015:49(02):31-3.

61. Wang P. Therapeutic effect of combined Western and Chinese medicine on intrahepatic cholestasis in pregnancy. J Nantong Univ (Medical Sciences). 2014;34(4):327-8.

62. Wang LX, Han LW, Wei DD. Effect of Yinzhihuang granules on glycolipid metabolism in the patients with intrahepatic cholestasis during gestation period and the concentration changes of MRP in placental bile salts transporters. World Chin Med. 2018;13(08):1871-4.

63. Wei FF. Self-prepared Modified Yinchen Decoction combined with ursodeoxycholic acid for intrahepatic cholestasis during pregnancy. Pract Clin J Integr Tradit Chin Med. 2016;16(9). https://doi.org/10.13638/j.issn.16714040.2016.09.014.

64. Zhang YH. Evaluation of clinical effect of ursodeoxycholic acid combined with Yinzhihuang oral liquid I treatment of intrahepatic cholestasis of pregnancy. China Med Pharm. 2017;7(07):193-5.

65. Zheng LC. Clinical observation of Lidan decoction combined with medicine in the treatment of intrahepatic cholestasis syndrome during pregnancy. Clin J Chin Med. 2019;11(18):135-7.

66. Gu Y, Xu Y, Gu P-j, He J. Clinical observation on Chinese medicine for the treatment of intrahepatic cholestasis of pregnancy. Shanghai J Tradit Chin Med. 2014;48(7)

67. Mao XY, He XX. Therapeutic effect of combined Western and Chinese medicine on intrahepatic Cholestasis in pregnancy:118 cases. Zhejiang J Integr Tradit Chin Med. 2014;24(5):445-6.

68. Liu M. Effect of Yinchenhao decoction on estrogen level in intrahepatic cholestasis during pregnancy. Sichuan Zhongyi. 2016;3:94-5.

69. Zhu NN, Huang SB. Therapeutic effect of combined Western and Chinese medicine on intrahepatic Cholestasis in pregnancy. J Sichuan Tradit Chin Med. 2008;26(8):83-4.

70. Liu Y-n, Wu R-x, Huang R-I. Clinical study on Wuling pills combined with ademetionine in treatment of intrahepatic cholestasis of pregnancy. Drugs. 2019;34(6):1771-3.

71. Wang SP, Chen BY, Liu ZH, et al. Clinical efficacy of oriental virgate wormwood decoction combined with S-adenosyl methionine in treatment of intrahepatic cholestasis of pregnancy and effect on Th17/Treg balance. J New Med. 2015;46(5):308-11.

72. Aiping D, Li Y. Clinical observation of Yi-guan-decoction combined with ursodeoxycholic acid in the treatment of intrahepatic cholestasis during pregnancy. Chin J Integr Tradit Western Med Liver Dis. 2014;000(003):148-9. https://doi.org/10.3969/j.issn.1005-0264.2014.03.008.
73. Zhang JC, Luo M. Treatment of combined blood-raising and wind-dispelling method and SAMe for intrahepatic cholestasis of pregnancy. Chongqing Med. 2005;34(04):583-4.

74. Nong ZF, Wu YW, Li WW, Wang DY, Gong YL. Systematic review and metaanalysis of integrative treatment on neonatal jaundice. J Liaoning Univ Tradit Chin Med. 2013;15(03):130-3.

75. Yan JJ, Xia XP, Bu N. Meta-analysis of Yinzhihuang oral liquid in treatment of intrahepatic cholestasis of pregnancy. China J Chin Materia Medica. 2016; 41(23):4428-35.

76. Suo ZM, Chen M. Meta-analysis of randomized controlled trials in using Yinchenhao decoction for the treatment of neonatal jaundice. J Liaoning Univ Tradit Chin Med. 2017;19(04):135-13.

77. $\mathrm{Wu} Y \mathrm{~L}$, Li ZL, Zhang XB, et al. Yinchenhao decoction attenuates obstructive jaundice-induced liver injury and hepatocyte apoptosis by suppressing protein kinase RNA-like endoplasmic reticulum kinase-induced pathway. World J Gastroenterol. 2019;25:6205-21.

78. Chen Z, Ma X, Zhao Y, et al. Yinchenhao decoction in the treatment of cholestasis: a systematic review and meta-analysis. J Ethnopharmacol. 2015; 168:208-16.

\section{Publisher's Note}

Springer Nature remains neutral with regard to jurisdictional claims in published maps and institutional affiliations.
Ready to submit your research? Choose BMC and benefit from:

- fast, convenient online submission

- thorough peer review by experienced researchers in your field

- rapid publication on acceptance

- support for research data, including large and complex data types

- gold Open Access which fosters wider collaboration and increased citations

- maximum visibility for your research: over $100 \mathrm{M}$ website views per year

At BMC, research is always in progress.

Learn more biomedcentral.com/submissions 